ARTICLE

\title{
Cell segmentation-free inference of cell types from in situ transcriptomics data
}

\author{
Jeongbin Park ${ }^{1,2,3,10}$, Wonyl Choi ${ }^{4,10}$, Sebastian Tiesmeyer ${ }^{1}$, Brian Long (10 ${ }^{5}$, Lars E. Borm ${ }^{6}$, Emma Garren ${ }^{5}$, \\ Thuc Nghi Nguyen (10 ${ }^{5}$, Bosiljka Tasic (1) ${ }^{5}$, Simone Codeluppi (10) ${ }^{6}$, Tobias Graf ${ }^{1}$, Matthias Schlesner (1) ${ }^{7}$, \\ Oliver Stegle ${ }^{3,8}$, Roland Eils (10 ${ }^{1,9,11 凶}$ \& Naveed Ishaque (i) ${ }^{1,11 凶}$
}

Multiplexed fluorescence in situ hybridization techniques have enabled cell-type identification, linking transcriptional heterogeneity with spatial heterogeneity of cells. However, inaccurate cell segmentation reduces the efficacy of cell-type identification and tissue characterization. Here, we present a method called Spot-based Spatial cell-type Analysis by Multidimensional mRNA density estimation (SSAM), a robust cell segmentation-free computational framework for identifying cell-types and tissue domains in 2D and 3D. SSAM is applicable to a variety of in situ transcriptomics techniques and capable of integrating prior knowledge of cell types. We apply SSAM to three mouse brain tissue images: the somatosensory cortex imaged by osmFISH, the hypothalamic preoptic region by MERFISH, and the visual cortex by multiplexed smFISH. Here, we show that SSAM detects regions occupied by known cell types that were previously missed and discovers new cell types.

\footnotetext{
${ }^{1}$ Berlin Institute of Health at Charité - Universitätsmedizin Berlin, Digital Health Center, Kapelle-Ufer 2, 10117 Berlin, Germany. ${ }^{2}$ Faculty of Biosciences, Heidelberg University, Heidelberg, Germany. ${ }^{3}$ Division of Computational Genomics and System Genetics, German Cancer Research Center (DKFZ), Heidelberg, Germany. ${ }^{4}$ Department of Computer Science, Boston University, Boston, MA, USA. ${ }^{5}$ Allen Institute for Brain Science, Seattle, WA, USA. ${ }^{6}$ Division of molecular neurobiology, Department of medical biochemistry and biophysics, Karolinska Institutet, Stockholm, Sweden. ${ }^{7}$ Bioinformatics and Omics Data Analytics, German Cancer Research Center (DKFZ), Heidelberg, Germany. ${ }^{8}$ Genome Biology Unit, European Molecular Biology Laboratory (EMBL), Heidelberg, Germany. ${ }^{9}$ Health Data Science Unit, Heidelberg University Hospital, Heidelberg, Germany. ${ }^{10}$ These authors contributed equally: Jeongbin Park, Wonyl Choi. ${ }^{11}$ These authors co-supervised this work: Roland Eils, Naveed Ishaque. ${ }^{{ }}$email: roland.eils@bih-charite.de; naveed.ishaque@bih-charite.de
} 
T he underlying transcriptional and spatial heterogeneity of cells gives rise to the plethora of phenotypes observed in cell types, tissues, organs, and organisms. Recent technological advances $^{1}$ have seen the profound adoption of single-cell sequencing to unravel transcriptional heterogeneity in healthy and diseased tissues, and have subsequently given rise to international consortia such as the human cell atlas (HCA) $)^{2}$. Such efforts would not be possible without computational frameworks supporting the analysis of single-cell sequencing data ${ }^{3}$. Linking this transcriptional heterogeneity with spatial heterogeneity of cells is a critical factor in understanding cell identity in the context of the tissue, for example, revealing the transcriptional basis of invasive cancer regions ${ }^{4}$ and highlighting the rich diversity of neuronal subtype expression and localization ${ }^{5}$. Recently developed multiplexed fluorescence in-situ hybridization ${ }^{6-8}$ and in situ mRNA tissue sequencing techniques ${ }^{9-14}$ have enabled the simultaneous measurement of multiple mRNAs in a spatial context.

Traditionally, mRNA molecules identified by in situ transcriptomics are assigned to cells and subsequently used for computing gene expression profiles of those cells ${ }^{15-18}$. Identification of cells relies on cell segmentation, a procedure demarcating the interior and exterior of the cell membranes, which relies on additional signals or landmarks obtained by staining nuclei ${ }^{19}$, cell membrane ${ }^{20-22}$, or total poly-A RNA ${ }^{5,6}$. However, accurate cell segmentation is difficult to achieve with current techniques due to tightly apposed or overlapping cells, uneven cell borders, varying cell and nuclear shapes, signal intensity variation, probe fluorescence emission efficiency variation, and tiling artifacts ${ }^{23}$. Such obstacles can result in detecting fewer cells or incorrect cell borders. Subsequent analysis would then be spatially restricted to inaccurately segmented cells and may mean that large portions of meaningful mRNA signals are discarded. This may result in incorrect celltype signatures, incomplete cell-type maps, or missing rare cell types. Therefore, there is a need for robust cell segmentationindependent methods for identifying cell-type signatures, celltype organization, and tissue domains from multidimensional mRNA expression data in complex tissues. These methods could be used for datasets lacking landmarks or to validate segmentation-based approaches.

In this work, we introduce a computational framework named spot-based spatial cell-type analysis by multidimensional mRNA density estimation (SSAM). In contrast to existing methods, SSAM departs from the spatial restriction of approaches based on cell segmentation and instead identifies cell types using mRNA signals in the image, without the need for prior cell segmentation. Furthermore, instead of labeling only segmented regions, our approach assigns cell-type labels to each pixel, ensuring a more complete picture of cell-type specific spatial heterogeneity. We apply SSAM to three mouse brain tissue images obtained by different techniques: the somatosensory cortex (SSp) by osmFISH, the hypothalamic preoptic region (POA) by MERFISH, and the visual cortex (VISp) by multiplexed smFISH. With all three datasets, we demonstrate the robustness of SSAM in identifying (1) cell types in situ, (2) spatial distribution of cell types, (3) spatial relationships between cell types, and (4) tissue domains (e.g., cortical layers) based on the local composition of cell types without fine-tuning of parameters. We demonstrate that SSAM (1) correctly identifies the spatial distribution of known cell types in regions missed in the SSp by cell segmentation based methods for the osmFISH data; (2) can analyze the POA MERFISH 3D data using the same parameters as for the 2D SSp osmFISH data without any extra adjustments of the settings; (3) identifies new and rare cell types in the VISp multiplexed smFISH data.

\section{Results}

The SSAM computational framework. SSAM consists of 4 major steps (Fig. 1), namely (1) mRNA signal estimation and downsampling; (2) computation of cell-type signatures; (3) generation of a cell-type map; and (4) identification of tissue domains.

In the first step, SSAM estimates mRNA signal intensity over the tissue image (Fig. 1A). Firstly, for each gene, mRNA signal intensity distribution is estimated by applying a Kernel Density Estimation (KDE) with a Gaussian kernel, which is then resolved to pixels in the image. The mRNA signal intensity distribution for each gene is stacked to create a gene expression vector field, which is a multichannel image where the pixels encode the expected density of mRNA count for each gene. This essentially assigns gene expression profiles to pixels in the image.

In the second step, SSAM identifies cell-type gene expression signatures by clustering (Fig. 1B). Before running the clustering algorithm, SSAM downsamples gene expression vectors to reduce computational processing time. As default, SSAM performs informed downsampling by selecting pixels that are local maxima in the gene vector field (Methods). After that, both the downsampled vectors and the gene expression vector field are normalized (Methods). SSAM clusters the sampled vectors using either DBSCAN ${ }^{24}$, HDBSCAN ${ }^{25}$, OPTICS $^{26}$, or the Louvain community detection method implemented in Seurat $^{27}$ (Methods). The Louvain methods is the default as it has been widely utilized to analyze single cell data. After the clustering step, sampled vectors with a large distance in gene expression space to their cluster medoid are removed as outliers to ensure the quality of selected vectors (Supplementary Fig. 1B). After that, each L1 maxima is assigned a unique cell-type label. The gene expression cluster centroids are used to represent the gene expression signature of a cell type.

In the third step, SSAM classifies each pixel in the image to create a 'cell-type map' (Fig. 1C, Supplementary Fig. 2A). SSAM includes a guided mode, which assigns pixels to a labeled set of given gene expression signatures (e.g. from scRNA-seq/segmentation), as well as a de novo mode, which assigns pixels to the cell type signatures obtained in the previous clustering step.

For the classification of pixels, SSAM first creates signature prototypes by averaging the signatures per cell-type class of the given signatures, then it classifies all pixels in the vector field according to the maximum correlation to any of the signature prototypes.

In the fourth step, SSAM identifies tissue domains that have distinct cell-type composition (Fig. 1D). SSAM computes the celltype compositions in a circular (or spherical) sliding window over the cell-type map and clusters the cell-type composition of each window using agglomerative hierarchical clustering (Supplementary Fig. 2B). The resultant clusters represent putative tissue domains. Clusters with high mutual correlation are then merged into a single tissue domain signature, and the cell-type composition of each domain is calculated.

In the following sections, we apply SSAM to three multiplexed FISH datasets obtained using different techniques. We reanalyze two previously published datasets, profiled by osmFISH ${ }^{6}$ and MERFISH ${ }^{5}$, to demonstrate SSAM's strength in comparison to earlier methods. For a newly generated multiplexed smFISH dataset we demonstrate that SSAM can unravel novel biological insights into the spatial cellular organization of the brain.

Improved astrocyte and ventricle detection in the mouse SSp. To demonstrate the utility of SSAM, we analyzed published osmFISH data, where the transcripts of 33 cell-type marker genes were localized in 2D space of the mouse brain somatosensory cortex $(\mathrm{SSp})^{6}$ (Figs. 2 and 3, Supplementary Figs. 3 and 4). We 


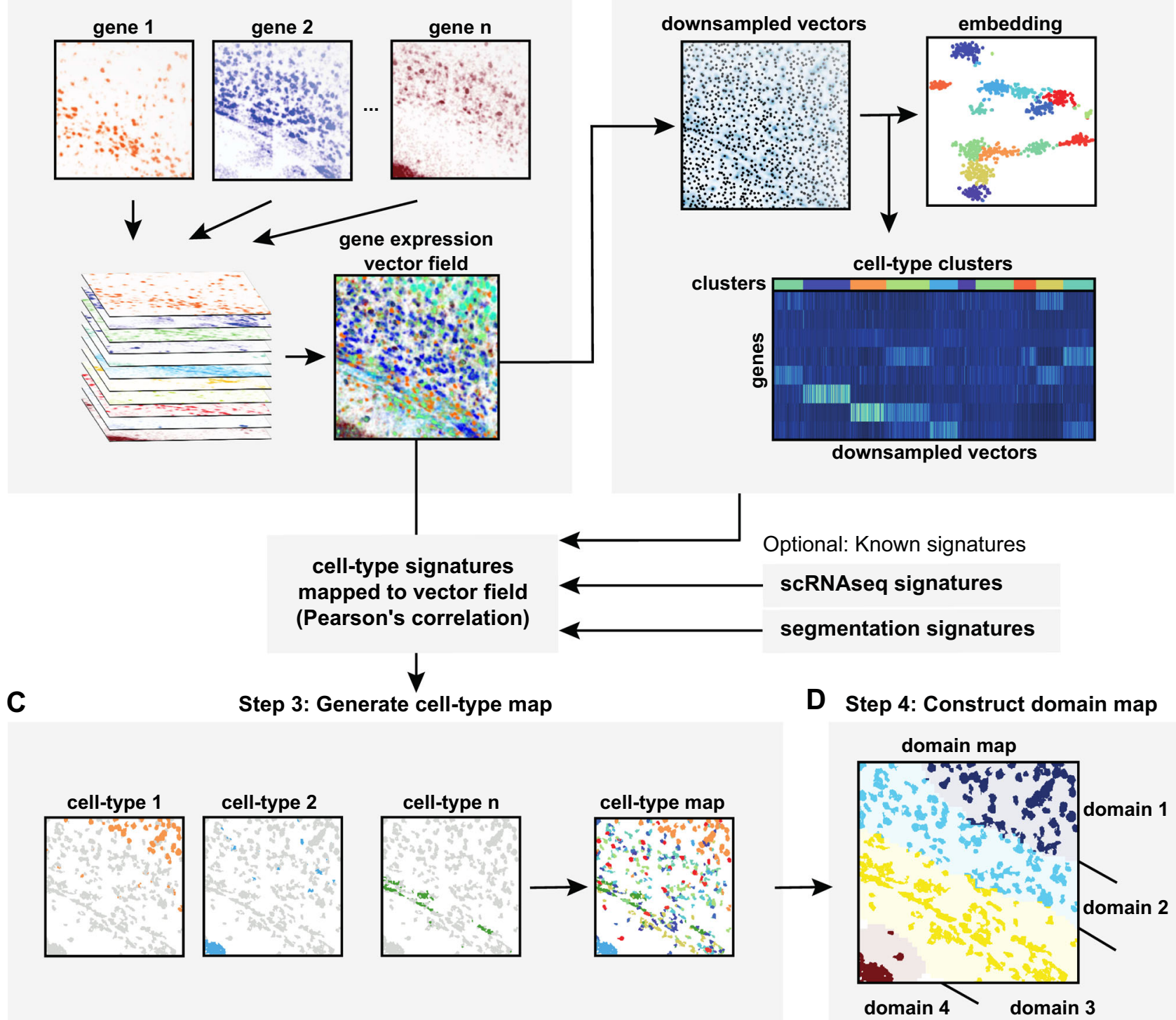

Fig. 1 Schematic diagram of the SSAM computational workflow for cell type and tissue domain definition based on gene expression data. A In step 1 , SSAM converts mRNA locations into a vector field of gene expression values. For this, SSAM applies a Gaussian KDE to mRNA locations for each gene and projects the resulting mRNA density values to pixels that represents coordinates in the tissue. The mRNA density estimated per each gene is stacked to produce a 'gene expression vector field' over the image. The gene expression vector field is analogous to a 2D/3D image where each pixel/voxel encodes the averaged gene expression of the unit area. Further details of the application of KDE can be found in Supplementary Fig. 1A; B In step 2, cell-type signatures are identified de novo. First, the gene expression profile at probable cell locations is identified as the local regions in the gene expression vector field where the signal is highest. These downsampled gene expression signals are identified and used for de novo cell type identification by cluster analysis. Alternatively, previously defined cell-type signatures can be used. C In step 3, a cell-type map is generated. For this, the cell-type signatures are mapped onto the gene expression vector field and cell types are assigned based on Pearson's correlation between each cell-type expression signature to the vector field to define cell-type distribution in situ. Further details about creating the cell-type map can be found in Supplementary Fig. 2A; D In step 4, the tissue domains are identified. The tissue domain signatures are identified using a sliding window to compute domain signatures based on the count of cell-type labels in the window. The tissue domains are defined by clustering these signatures. Further details on creating the tissue domain map can be found in Supplementary Fig. 2B.

compare results obtained from SSAM against the results obtained from Poly-A segmentation from the original study.

The osmFISH dataset was first analyzed using the guided mode of SSAM. Cell-type maps were generated using cell-type signatures from the prior segmentation-based approach ${ }^{6}$ and another from scRNA-seq ${ }^{28,29}$ (Supplementary Fig. 4E).

To quantify the similarity between the prior segmentation and the cell-type maps generated by SSAM, we calculated a 'matching score' for each cell type (Methods). The matching scores between the segmentation from the previous study and SSAM guided by both segmentation-based and scRNA-seq cell-type signatures were generally high (mean and median matching score of 0.67 and 0.78 for segmentation-based, 0.60 and 0.70 for scRNA-seqbased signatures, respectively), indicating a strong agreement of the two cell-type maps as visually apparent (Supplementary Tables 1 and 2, Supplementary Figs. 5 and 6). 
A

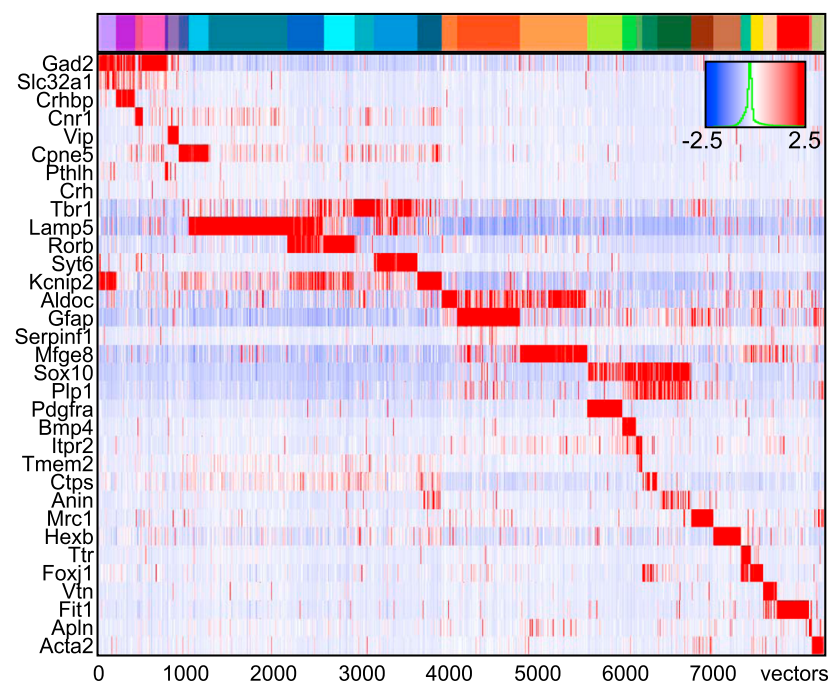

B

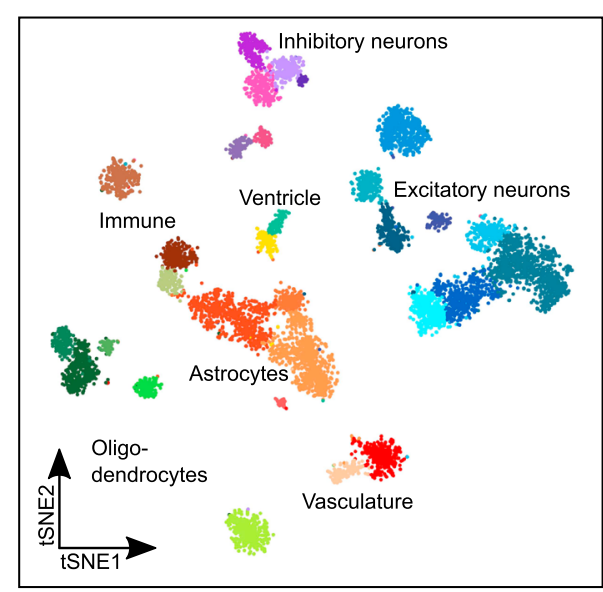

D

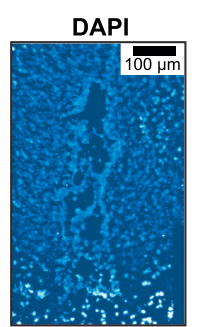

Foxj1

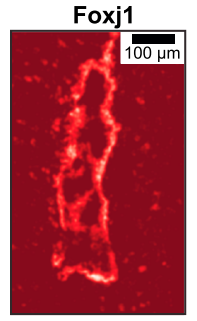

PolyA

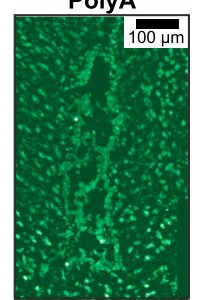

Ttr

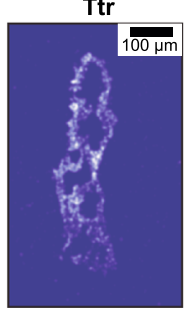

I Inhib. Kcnip

- Inhib. Čnr1

- Inhib. Rest

Inhib. Vip

Py. Cpne5

Py. L2-3 L2

- Py. L3-4

- Py. $L 5$

- Hippocampus

- Astro. Aldoc

- Astro. Gfap

OPC

- OD COP

- OD MF

- OD Mature

1 - PVM

1 C. Plexus

1 Pericytes

- Endothelial
Endothelial 1
VSM

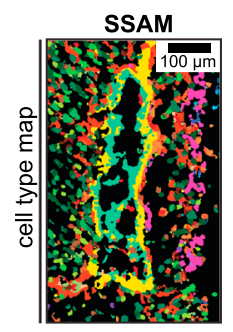

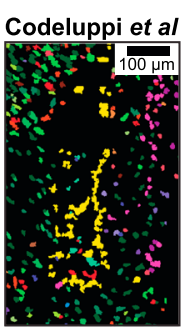
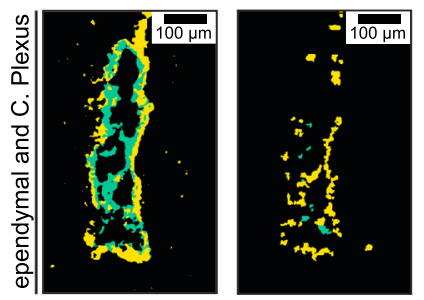

C

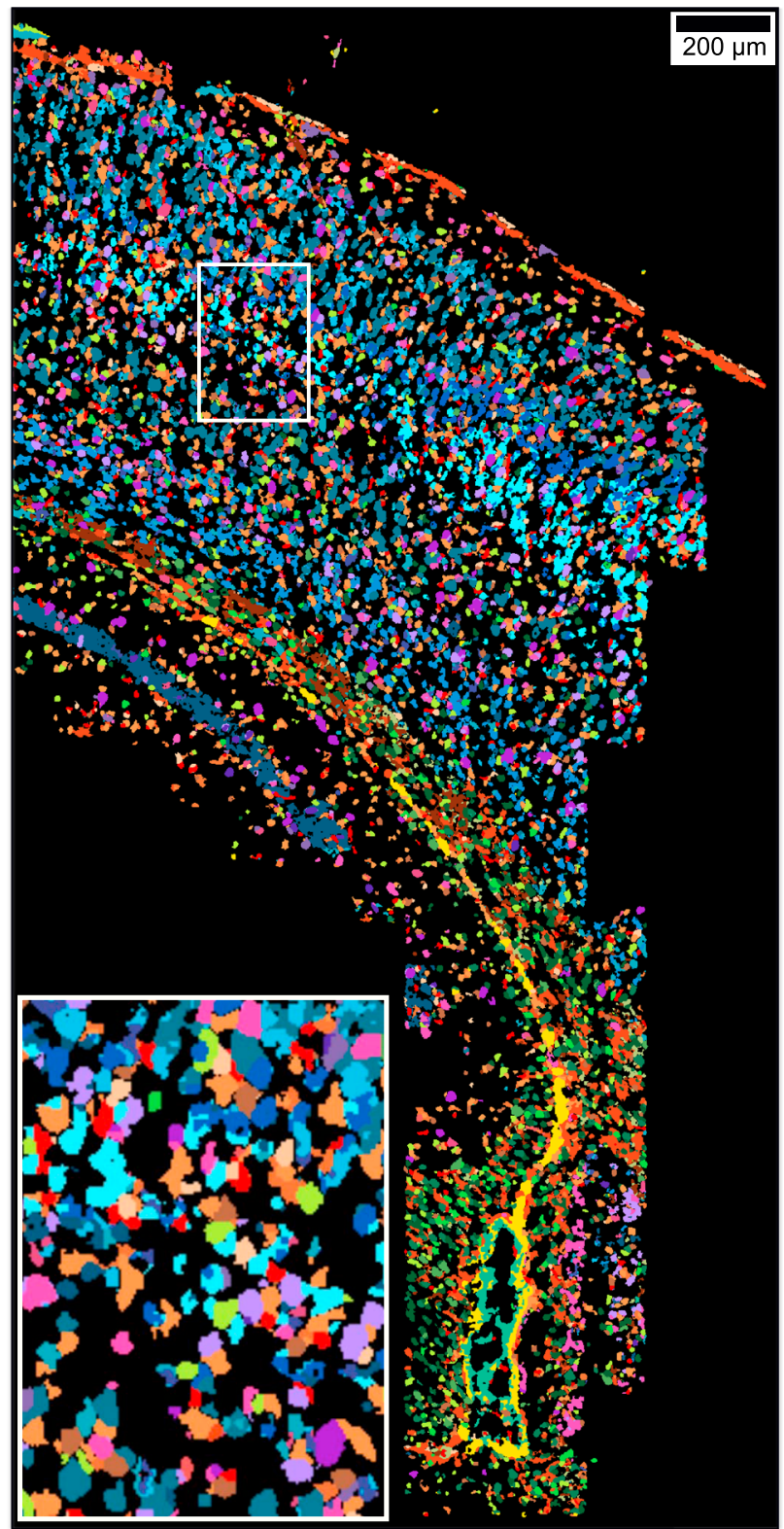

$E$

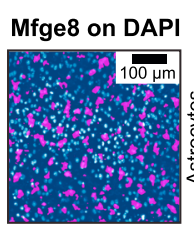

Mfge8 on PolyA

PolyA
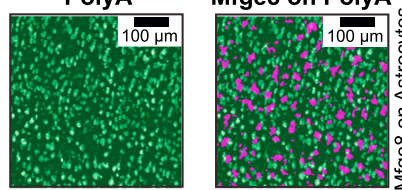

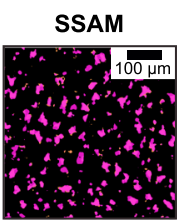

Codeluppi et al
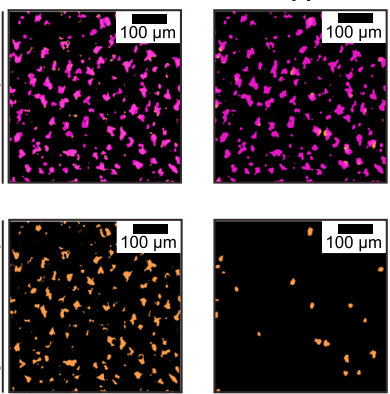

Next, we continued with completely de novo cell-type identification. The resulting 30 cell-type signatures (Fig. 2A, B, Supplementary Figs. 7-10) were consistent with those identified in the segmentation-based clustering and scRNA-seq based cell-type signatures $^{6}$ (Supplementary Fig. 4C, D), implicating the robustness of the de novo cell-type calling by SSAM. Each of the SSAM de novo cell-type signature clusters were assigned the label of the closest correlating segmentation-based cluster.

As with the guided mode analysis, we limit the comparison to the most comparable cell types, excluding cell types with low 
Fig. 2 SSAM improves astrocyte and ventricle detection in the mouse SSp region. A Gene expression heatmap showing cell-type-specific expression of marker genes (8,252 vectors). Rows show z-scored gene expression and columns show the gene expression patterns of filtered local maxima vectors. The top annotation shows the cell types and coloring based on the best correlating segmentation-based cell-type signature from Codeluppi et al. The colors of the top annotation correspond to the cell type legend in Fig. 2B; B A t-SNE map of cell-type signatures with distinct expression. Cell-type clusters are visualized as a 2D t-SNE embedding of filtered local maxima vectors. Cell-type annotation and coloring are based on the best correlating segmentationbased cell-type signature from Codeluppi et al (Supplementary Fig. 4C, D). The cell-type legend is grouped by cell-type classes labels shown in the t-SNE plot, and is based on groupings by Codeluppi et al.; C The SSAM de novo cell-type map showing the spatial organization of the cell types signatures in the gene expression vector field. Inset shows a zoom-in of the highlighted tissue region. The colors of the cell types correspond to the cell-type legend in B; D SSAM improves the reconstruction of the ventricle. The upper left two panels show the DAPI and Poly-A signal around the ventricle area, showing tightly packed cells (occlusion) and lower signal in the ventricle structure compared to surrounding cells. The lower-left two panels show the KDE gene expression signature for Foxj 1 (the marker for ependymal cells) and Ttr (the marker for choroid plexus cells). The bottom right two panels show the reconstructions of only the ependymal (yellow) and choroid plexus (teal) cell types by SSAM and Codeluppi et al.; E The far-left upper and lower panels show DAPI and PolyA signals. The middle left upper and lower panels show the overlap of the Mfge8 signal with DAPI and Poly-A signals. The top right two panels show the cell-type signals for Mfge8 expressing astrocytes by SSAM and Codeluppi et al. The bottom right two panels show the overlay of the Mfge8 signal with the cell-type calls by SSAM and Codeluppi et al.

correlation in gene expression signatures $(<0.8)$ (Supplementary Table 3, Supplementary Fig. 11). The matching score result showed high average values (mean and median of 0.76 and 0.83 , respectively) and $81 \%$ of cell types had a matching score of greater than 0.6. Comparing marker gene expression of cell types having lowest matching score $(<0.3)$ (Supplementary Table 3) confirmed that the SSAM guided cell-type map is in better agreement with their marker gene expression (Supplementary Figs. 12 and 13). Given the low correlation of C. Plexus cell type to the corresponding osmFISH cluster, which is one of the dominant cell types in the ventricle region, high-resolution investigation of Poly-A and DAPI signals confirm the existence of both cell types in the ventricle area (Fig. 2D). Since ependymal and choroid plexus cells were small and tightly packed and exhibit relatively lower DAPI and poly-A signal, we concluded that the performance of the watershed algorithm was insufficient to identify cells in the area. Furthermore, we statistically evaluated this for each cell type by comparing the gene expression in the unique parts of the segmentation and SSAM de novo cell-type map, to the overlapping parts (Methods). Gene expression of the unique part of SSAM de novo cell-type map showed higher correlation to the overlapping regions compared to the unique parts of the segmentation (Supplementary Fig. 14).

We then performed domain analysis on the SSAM de novo cell-type map. Identified domains correlated well with the known cerebral cortex layers, consistent with results reported in the previous study (Fig. 3A). Laminar distribution of cell types is established $^{30}$, and can be considered as a ground truth for validating the cell-type map. Cell-type assignments of excitatory pyramidal cells in the cortical layers conformed closely to known localizations (Supplementary Fig. 15). The domains identified as: layer 2/3 primarily consists of Pyramidal L2-3/L5, L2-3, and L3-4 cell types; layer 4 consists of Pyramidal L4 and L3-4 cell types; layer 5 consists of Pyramidal L3-5 and L5 cell types; and layer 6 consists of Pyramidal L6 cell types.

In addition, cell-type composition of the domains revealed that Mfge8 expressing astrocytes (Astrocyte Mfge8) contributed $7-14 \%$ of each of the tissue layers (Fig. 3B), in contrast to the significantly fewer numbers of Astrocyte Mfge8 cells called in the previous study ${ }^{6}$. Comparison of high-resolution images of DAPI and poly-A signals with Mfge8 expression densities implicates that the poly-A signal was not strong enough to discriminate the presence of astrocyte Mfge8 cells from the background, while the DAPI images clearly supported the existence of $M f g e 8$ expressing astrocytes at positions identified by SSAM (Fig. 2E). The clear DAPI signal but low poly-A signal for these astrocytes Mfge8 suggested that they have a lower mRNA content compared to other cells. We compared the total counts of mRNA molecules of astrocytes and other cell types from mouse brain scRNA-seq data $^{31}$ and found that astrocytes exhibited significantly less mRNA molecules than other cell classes (Supplementary Fig. 4B). Our observation reveals the inadequacy of the watershed segmentation algorithm applied to poly-A signal when not considering cells with a low total mRNA content. This implies that the original segmentation of these cell types could be less accurate than the SSAM de novo cell-type map, therefore also reducing the matching score for these cell types.

Diversity of neuronal cell types in the mouse POA in 3D. To demonstrate the performance of SSAM for three-dimensional in situ transcriptomics data, we applied SSAM to previously published MERFISH data, where 135 transcripts were localized in $3 \mathrm{D}$ space of the hypothalamic preoptic region (POA) of a mouse brain $^{5}$ (Fig. 4, Supplementary Figs. 16 and 18). We compare results obtained from SSAM against the results obtained from DAPI segmentation from the original study.

We applied both SSAM guided mode and de novo mode. For guided mode, the previously known cell-type signatures obtained by segmentation and scRNA-seq were used. For both guided and de novo modes, SSAM analysis was performed in 3D space, generating a 3D cell-type map (Fig. 4B). The resulting cell-type maps on the $x$-y plane at the center of slice on the $\mathrm{z}$-axis (at $5 \mu \mathrm{m}$ ) were visually similar to the previous study (Supplementary Fig. 17G). SSAM cell-type signatures showed high expression of their marker genes (Supplementary Figs. 18-21) and a high correlation to the cell-type signatures from both the segmentationbased clusters and scRNA-seq clusters (Supplementary Fig. 17E, F). Among them, 7 inhibitory and 4 excitatory neuronal cell types showed very high correlation $(>0.8)$ to the segmentation-based neuronal signatures, and also showed distinctive tissue localization patterns (Fig. 4D, E), similar to those previously reported (Supplementary Fig. 22).

We then quantified the similarity of the SSAM cell-type maps with the cell segmentation by Moffitt et al. The SSAM guided mode cell-type map achieved high matching scores for comparable cell types (mean and median of 0.76 and 0.83 for segmentation-based, 0.88 and 0.94 for scRNA-seq-based signatures, respectively), with only 6 of 76 cell-types exhibiting a low matching score $(<0.3)$ for segmentation-based case (Supplementary Tables 4 and 5, Supplementary Figs. 23 and 24). Comparing the SSAM de novo cell-type map also yielded high matching scores (mean and median of 0.83 and 0.93 , respectively) (Supplementary Table 6, Supplementary Fig. 25), further validating the computational approach adopted by SSAM to identify de novo cell-type signatures and generating cell-type maps. One of the most notable differences in the SSAM cell-type 
A

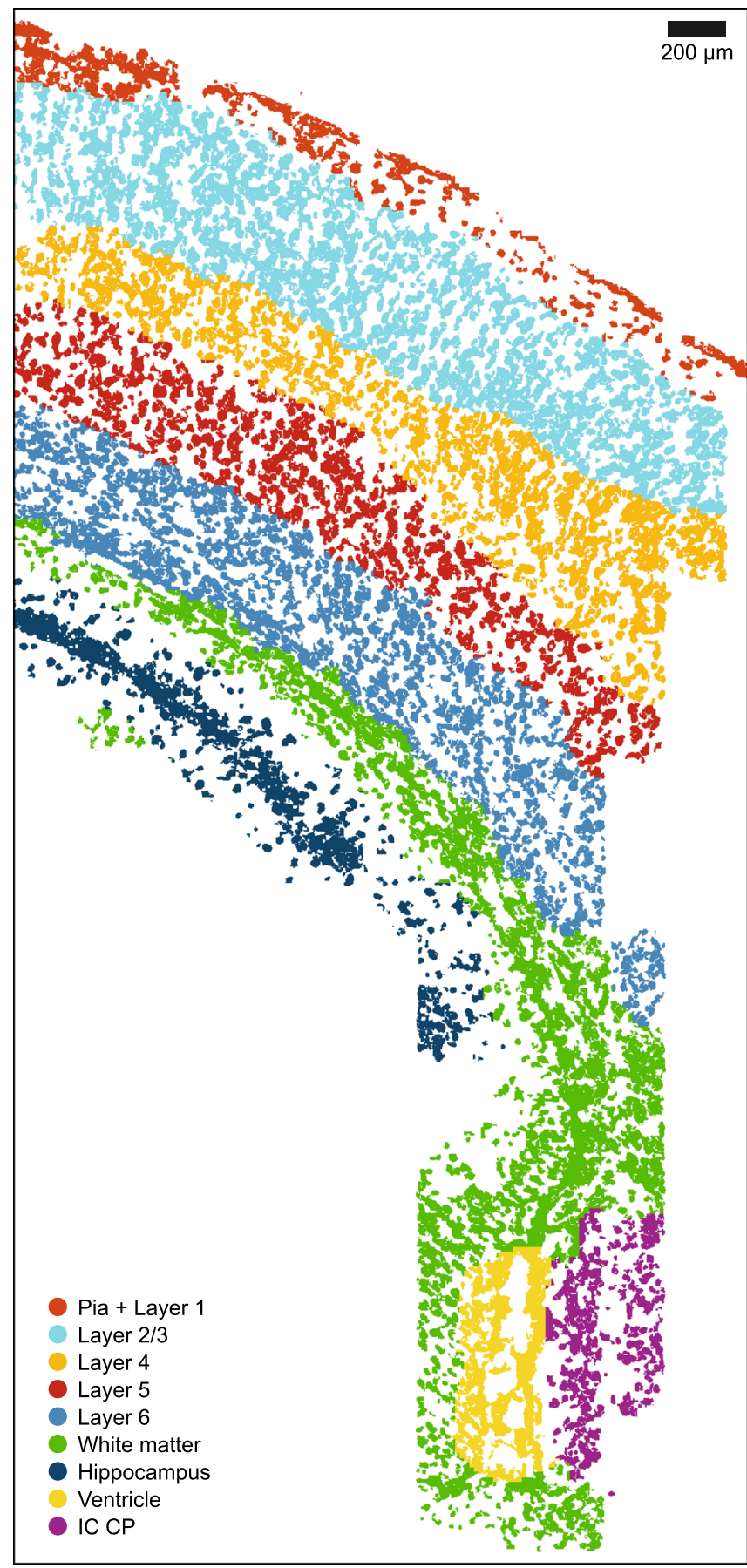

B

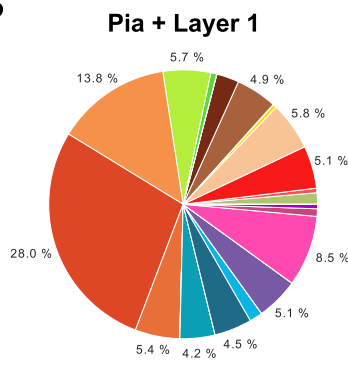

Layer 4
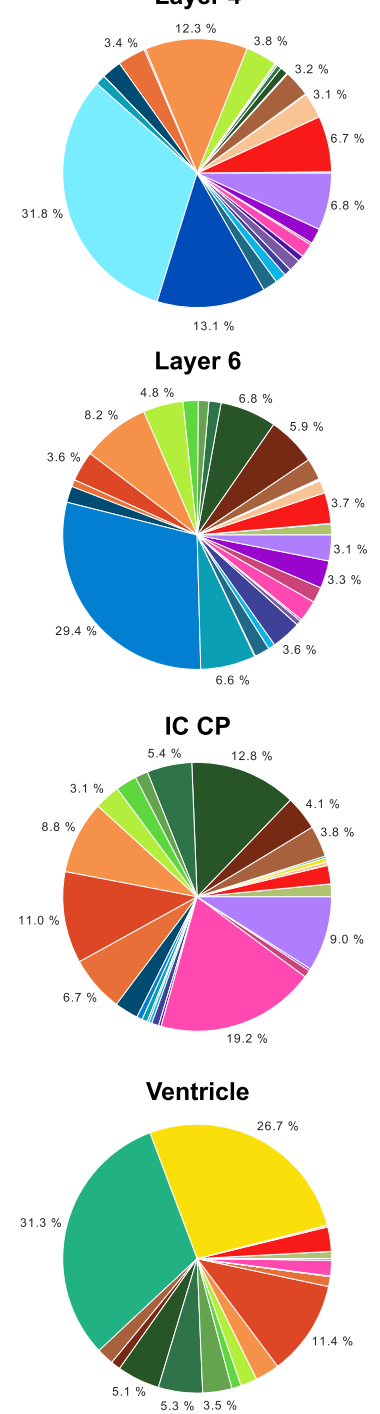

Layer 2/3

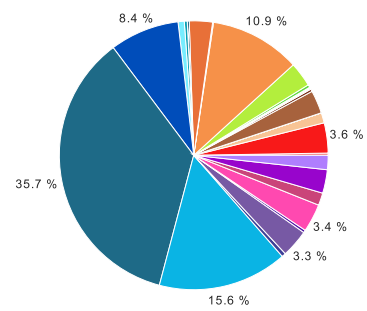

Layer 5

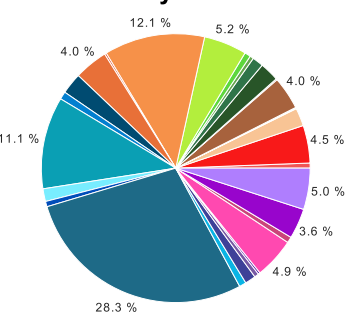

Hippocampus

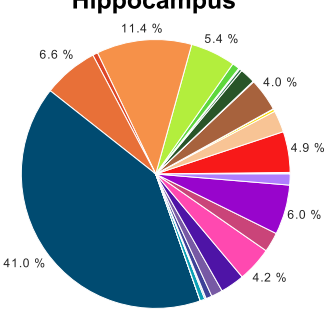

White matter

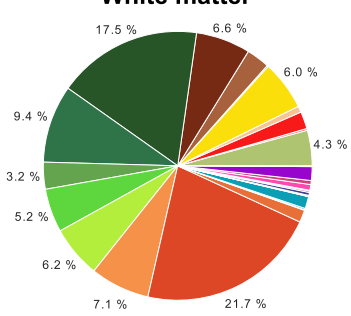

All

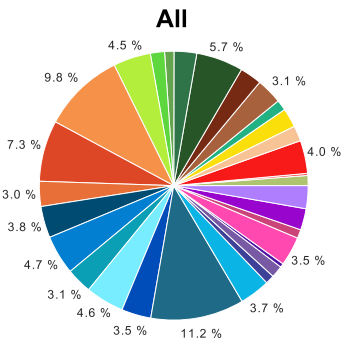

Fig. 3 SSAM identifies cortical layer tissue domains in the mouse SSp cortex. A Tissue domain map generated by SSAM. Tissue domain signatures were identified from clustering local cell-type composition over sliding $100 \mu \mathrm{m}$ circular windows and projected back onto the cell-type map. The reconstruction shows the various cortical layers; B Cell-type composition within each tissue domain. The plots show that each domain consists of 7-14\% Astrocyte Mfge 8 cell types, apart from the ventricle, which instead shows a majority of Choroid plexus and Ependymal cell types.

map was that we found a higher density of astrocytes compared to Moffit et al. A comparative analysis revealed that some astrocyte signals identified by SSAM were not found in the segmentation by Moffit et al. Note that the existence of astrocytes is clearly shown by the corresponding marker gene expression (Supplementary Fig. 26).

The generated tissue domain map identifies several domains consisting of regions consisting primarily of inhibitory neurons, excitatory neurons, and oligodendrocytes, as well as the ventricle structure (Supplementary Fig. 27).
Finally, we reconstructed a three-dimensional cell-type map (Supplementary Movie 1). While the thickness of the tissue image is limited $(10 \mu \mathrm{m})$, we demonstrate the shape and size difference of the whole cell-type map and the cell-type specific maps for inhibitory neurons, excitatory neurons, and astrocytes (Supplementary Movies 2, 3, 4).

Despite the difference in dimensionality between the osmFISH data (2D) and the MERFISH data (3D), SSAM was able to successfully process the data and produce meaningful results. More importantly, the analyzes in this section were performed 
A

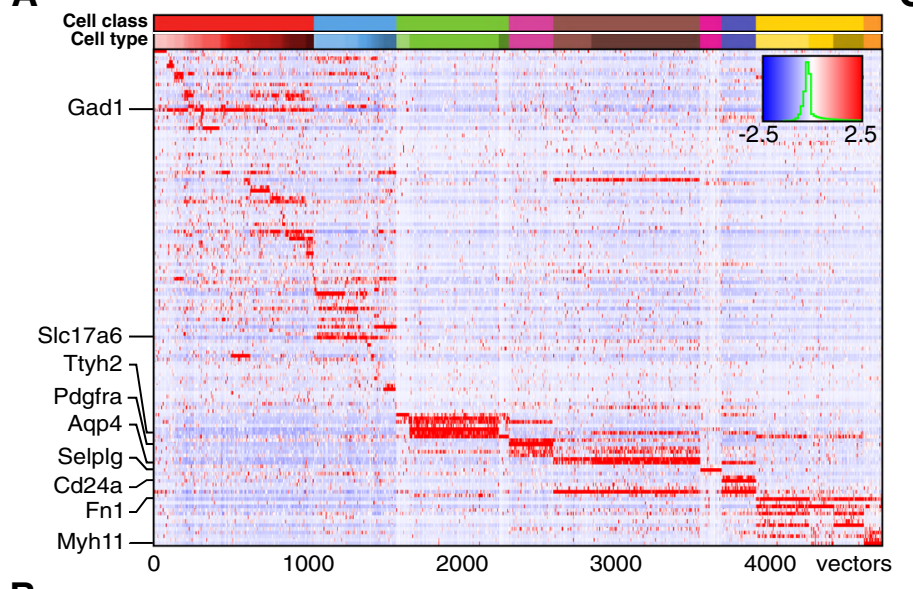

B

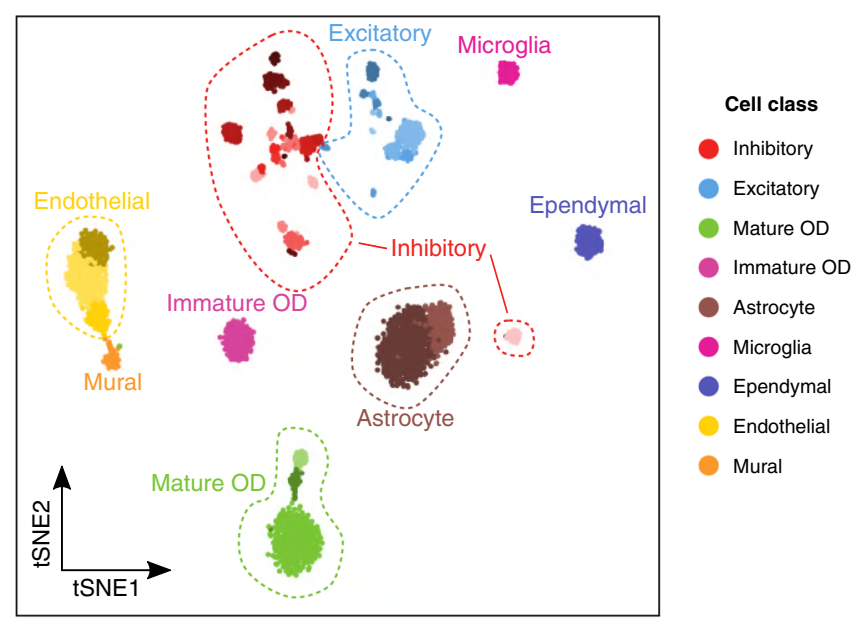

D

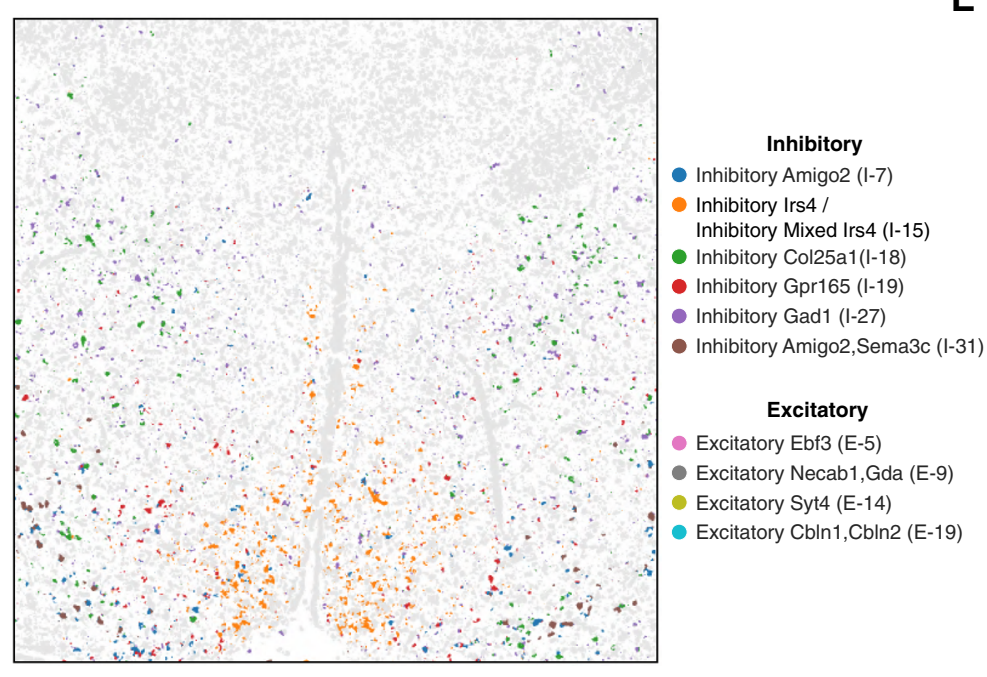

C
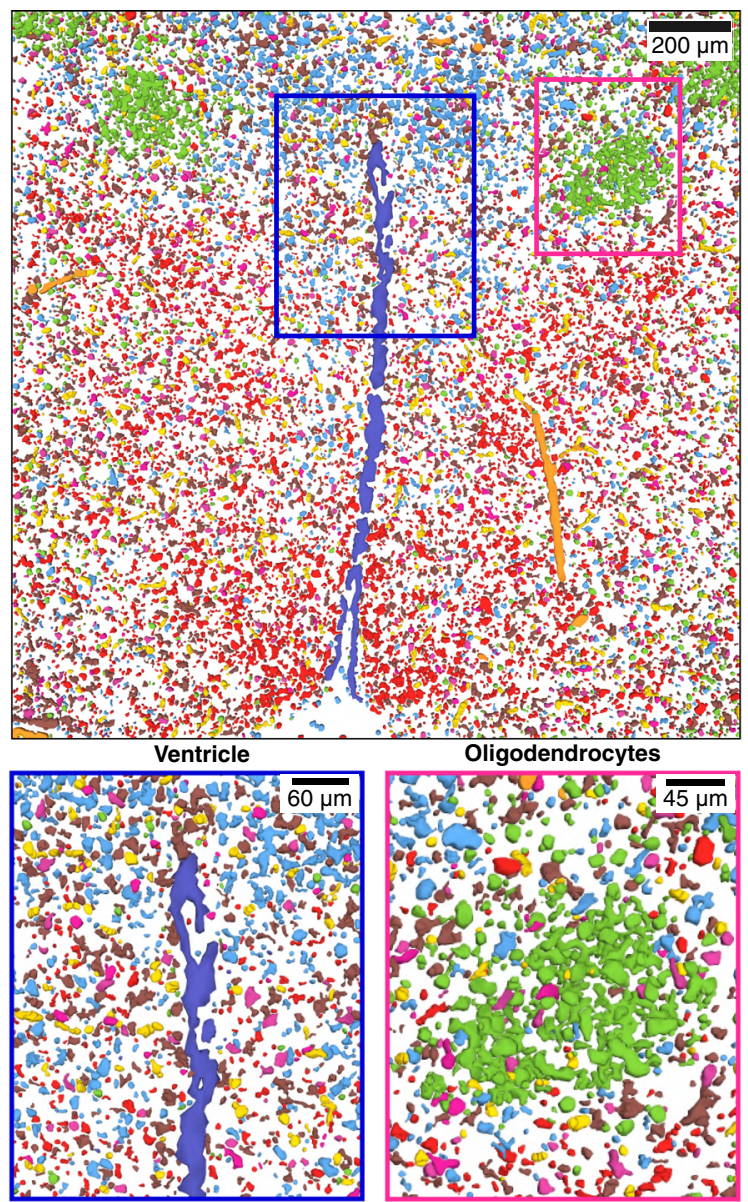

E

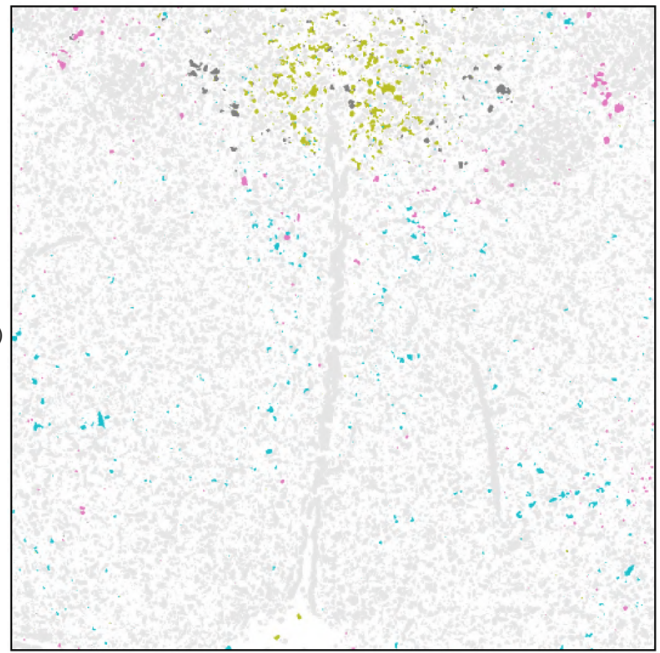

with almost the same procedure and parameters applied to the osmFISH data. Therefore, we set these parameters as the default values to facilitate rapid and robust analysis of other multidimensional in situ transcriptomics dataset using SSAM.

Identification of novel sub-layering in the mouse VISp. To further demonstrate that SSAM can be used for rapid and robust analysis of in situ transcriptomics data, we applied SSAM to unpublished multiplexed smFISH data of the mouse primary visual cortex (VISp) generated as part of the SpaceTx consortium $^{32}$ (Figs. 5 and 6, Supplementary Figs. 28 and 29). In total, the expression of 22 genes was quantified in situ (Methods).

Analysis of the tissue image was restricted to the manually defined VISp region (Supplementary Fig. 28D). SSAM was performed in both guided mode and de novo mode (Supplementary Fig. 29A). The guided mode of SSAM was performed using scRNA-seq data ${ }^{30}$. For the de novo run, the identified celltype signature clusters were assigned the label of the cluster in the scRNA-seq data with the highest correlation (Fig. 5A, B). Then, the tissue domains were identified based on the de novo cell-type 
Fig. 4 SSAM 3D cell-type map confirms rich diversity of heterogeneous cells in the posterior hypothalamic POA. A Gene expression heatmap showing cell-type-specific expression of marker genes (4,714 vectors). Rows show z-score normalized gene expression and columns show the gene expression patterns of filtered local maxima vectors. The bottom row of the top annotation shows the cell types. Due to a rich diversity of various inhibitory and excitatory neurons captured, the cell types were grouped into classes. The top row of the top annotation shows the cell classes which are named and colored based on the best cell-type signatures and cell classes from Moffitt et al. The colors of the cell classes in the top annotation correspond to the celltype legend in B. The colors of the cell types are available in Supplementary Fig. 16; B A t-SNE map of cell-type signatures with distinct expression. Celltype clusters are visualized as a 2D t-SNE embedding of filtered local maxima vectors. Cell-type annotation and coloring are based on the best correlating segmentation-based cell-type signature from Moffitt et al. The t-SNE map clearly shows the distinct cluster of different inhibitory and excitatory cell-type signatures. Cell types are grouped into classes based on groupings by Moffitt et al.; C The SSAM de novo 3D cell-type map showing the spatial organization of the cell types signatures in the gene expression vector field. Below left and right a zoom-in of the highlighted tissue regions of the ventricle structure and clusters of oligodendrocyte cell types. The colors of the cell types correspond to the cell-type legend in B; D Spatial localization of various inhibitory cell-type signatures. We found a number of inhibitory cell types that both matched expression signature and tissue localization described by Moffitt et al. See also Supplementary Fig. 22; E As D, but for excitatory cell types.

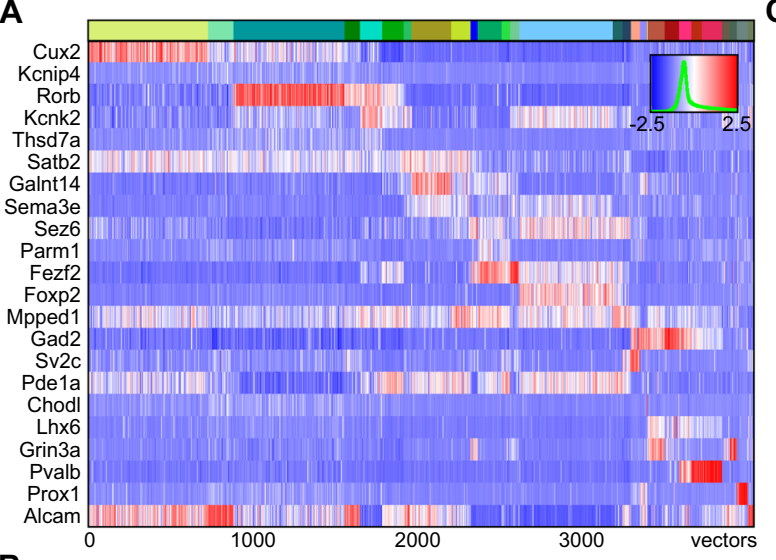

B

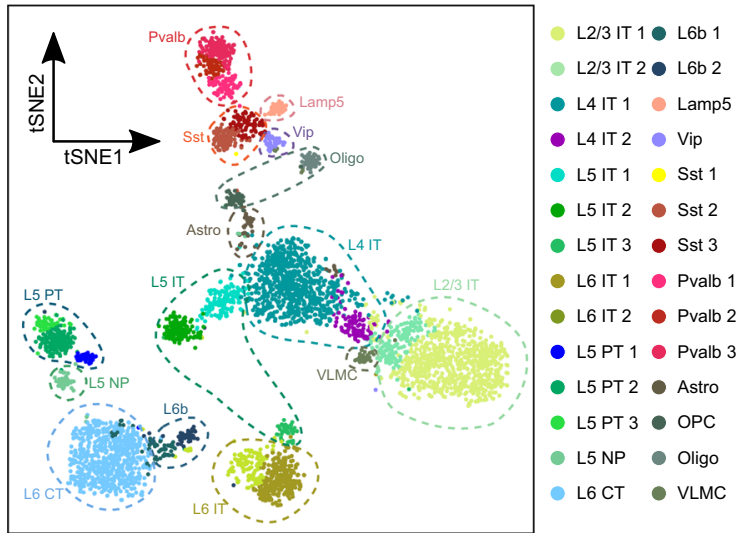

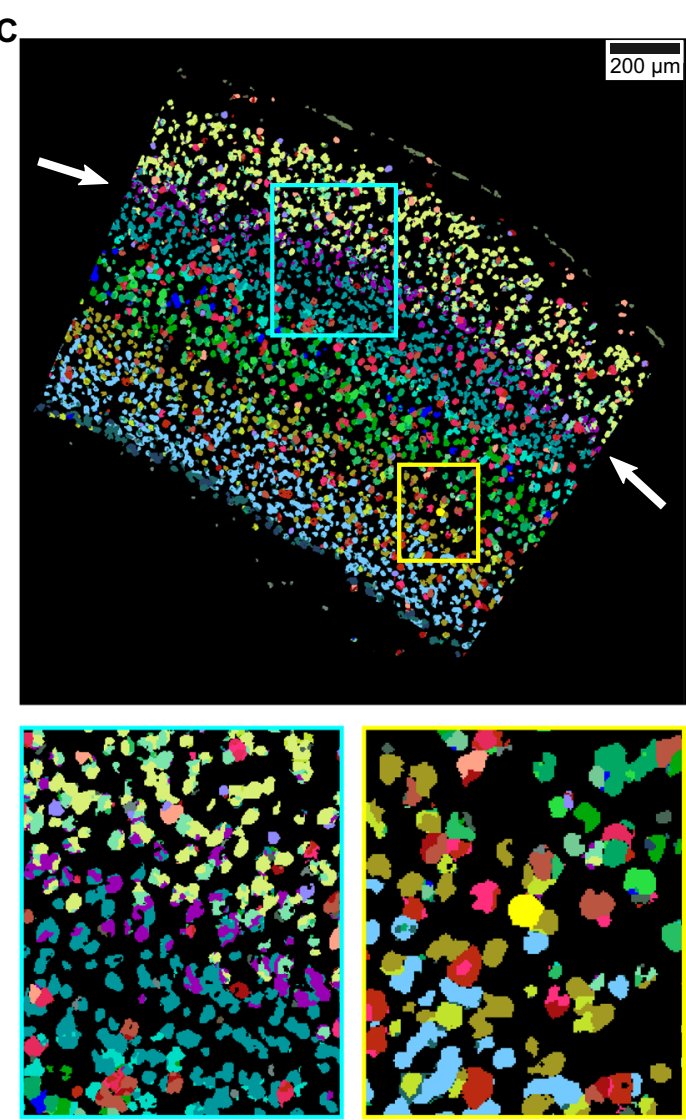

Fig. 5 SSAM identifies layer structure in VISp and confirms rare Sst Chodl cell type in the mouse VISp region. A Gene expression heatmap showing cell-type-specific expression of marker genes (4,113 vectors). Rows show z-score normalized gene expression and columns show the gene expression patterns of filtered local maxima vectors. The top annotation shows the cell types and coloring based on the highest correlating single-cell RNA-seq based cell-type signature from the previous result (Tasic et al., 2018). The colors of the top annotation correspond to the cell-type legend in B; B A t-SNE map of cell-type signatures with distinct expression. Cell-type clusters are visualized as a 2D t-SNE embedding of filtered local maxima vectors, with groupings based on the Supplementary Table 9 of Tasic et al. 2018. Cell-type annotation and coloring are based on the best correlating segmentation-based cell-type signature from the previous result (Tasic et al., 2018); C The SSAM de novo cell-type map showing the spatial organization of the cell types. Highlighted are the tissue regions of the cortex including novel L4 IT cell type sub-layering (main panel, purple, white arrows, lower left panel, see also Supplementary Fig. 29B), and rare Sst Chodl cell type (lower right panel, yellow, see also Supplementary Fig. 29C). The colors of the cell types correspond to the cell-type legend in $\mathbf{B}$.

map (Fig. 6), with the result showing the laminar structure of the VISp region. We identified two distinct layer 4 (L4) neuronal clusters. Interestingly, both of them showed the highest correlation to the single L4 IT type identified via scRNA-seq, but their spatial locations show a clear difference (Fig. 5C, Supplementary Fig. 29B). We named the cluster localizing to the superficial region of layer L4 as 'L4 IT Superficial' (L4 IT 2). This finding adds context to the previously observed heterogeneity of the L4 IT cell type $\mathrm{e}^{30}$, where we show that this heterogeneity determines superficial and deep localization in layer 4.

The cell-type map generated by SSAM guided mode were visually similar to that of de novo mode, except for the cell types found in the layer 2 (L2) (Supplementary Fig. 29A). We found that the majority of cell types found in L2 were assigned to the VLMC type in SSAM guided mode. We observed that this type was actually a neuronal type in L2. This cell type showed high 
A

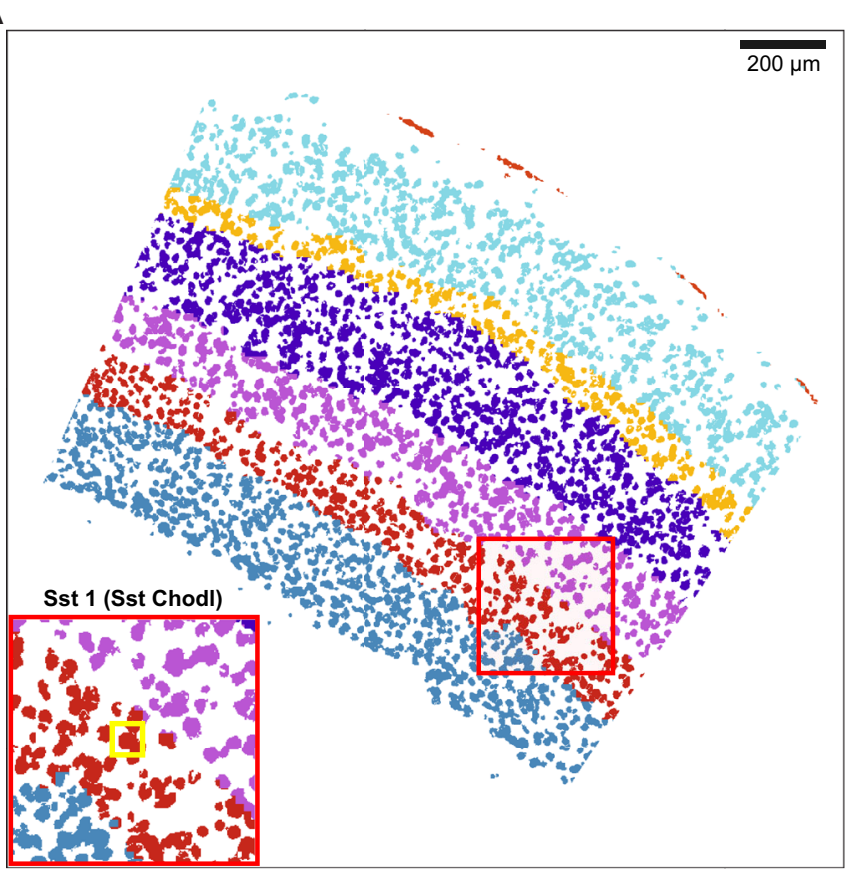

B
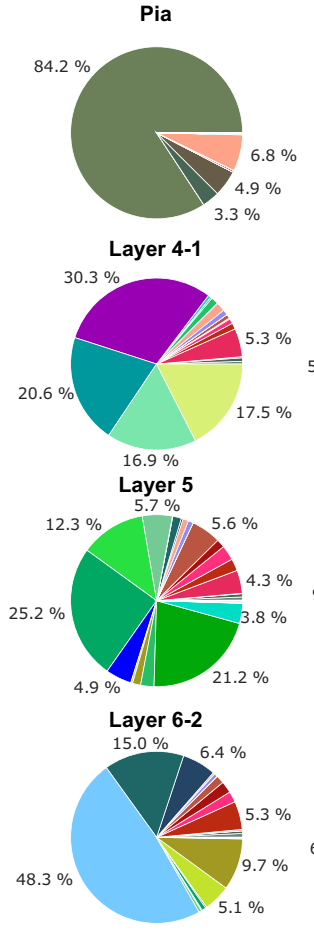

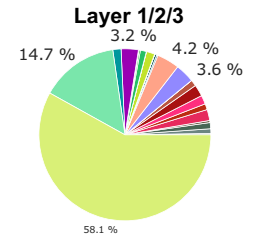

Layer 4-2
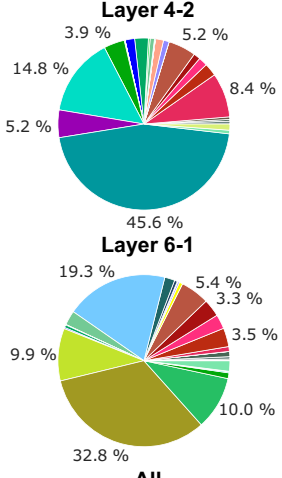

All

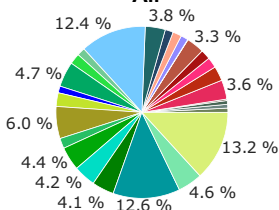

L2/3 IT 1

L2/3 IT 2

- L4 IT 1

L4 IT 2

L5 IT 1

L5 IT 2

L5 IT 3

- L6 IT 1

- L6 IT 2

- L5PT 1

- L5 PT 2

- L5 PT 3

- L5 NP

- L6 CT

- L6b 1

- L6b 2

Lamp5

- Vip

Sst 1

Sst 2

- Sst 3

- Pvalb 1

- Pvalb 2

- Pvalb 3

- Astro

- OPC

- Oligo

- VLMC

Pia Layer 1/2/3 Layer 4-1 Layer 4-2 Layer 5 Layer 6-1 Layer 6-2

Fig. $\mathbf{6}$ Rare Sst Chodl cell type localizes to the L6-1 layer of the mouse VISp region. A Tissue domain map generated by SSAM. Tissue domain signatures were identified from clustering local cell-type composition over sliding $100 \mu \mathrm{m}$ circular windows and projected back onto the cell-type map. The reconstruction shows the various cortical layers within the adult mouse VISp, with very clear separation of the Pia layer, and separation of layer 4 and layer 6 into 2 sub-layers. The inset zooms into the location of the rare Sst Chodl cell type found in layer 6-1; B Cell-type composition within each tissue domain.

expression of Alcam, a marker gene of the VLMC cell type, but low expression of other genes. Due to the limited number of genes profiled in the multiplexed smFISH experiment, lack of other neuronal marker genes led to incorrect high correlation of this type VLMC. However, SSAM properly assigned the centroid to be L2 neurons in de novo mode.

SSAM was also able to identify a rare cell type, Sst Chodl, which is known to be related to long-range projection and sleepactive neurons ${ }^{33-35}$. In addition, we mapped the Sst Chodl celltype signal to between layer L5 and L6 (Supplementary Fig. 29C), consistent with previously reported localization to L5 and $\mathrm{L}^{33}$. This finding was validated against its marker gene expression (Supplementary Figs. 30-32), and ultimately demonstrates SSAMs ability to identify cell-type signatures of lowly abundant and rare cell-types.

\section{Discussion}

We describe a segmentation-free computational framework for processing in situ transcriptomics data and demonstrate its performance on three different adult mouse brain datasets: the somatosensory cortex (SSp) profiled by osmFISH, the hypothalamic preoptic region (POA) by MERFISH, and the visual sensory cortex (VISp) by multiplexed smFISH. We find that the cell-type signatures and maps generated by SSAM for both osmFISH and MERFISH datasets were similar to the previously reported ones, validating the underlying methodology of SSAM. Based on this, we successfully determined cell types and constructed cell-type and tissue domain maps in the multiplexed smFISH mouse VISp dataset.

In the osmFISH dataset our method outperforms the original segmentation-based cell-type map reconstruction due to limitations in the segmentation process. In the MERFISH dataset we show that SSAM is able to identify diverse populations of cell types and that SSAM is scalable to $3 \mathrm{D}$ image data. For the VISp multiplexed smFISH dataset, SSAM identified a rare cell type and elucidated a suspected spatial heterogeneity of cell types in the cortex without segmenting a single cell. Overall, the results show that SSAM is not only a robust tool to validate segmentationbased methods, but also a reasonable alternative when segmentation is difficult or DAPI or Poly-A images are lacking.

However, for some questions it is important to distinguish between cells to e.g. delineate growth arising from increasing cell size vs cell proliferation or to investigate multinucleation in cardiomyocytes or cytotrophoblast cells. In cases such as these, we recommend the use of SSAM as a complementary method to segmentation-based analysis in two ways. First, the output of SSAM can be compared to validate that the segmentation process did not introduce artifacts. Secondly, to use the SSAM output as an input for the segmentation process to refine the segmentation procedure for different domains or cell-type signals.

In terms of methodological parsimony, SSAM minimizes the number of assumptions, avoids iterative optimization, and thus offers maximal transparency, interpretability, and reproducibility. The lightweight nature of the algorithm typically brings a considerable runtime advantage over other available packages. SSAM is written as a Python library, with some core analysis functions wrapped up with external $C$ functions to speed up the computation. The package is available as an easily installable Python package, and can easily be extended with existing in situ transcriptomics pipelines, e.g. starfish (https:/github.com/spacetx/ starfish) or Giotto ${ }^{36}$. SSAM is accompanied with a notebook outlining all the steps presented in this paper. Taken together, we present a flexible and robust method for fully automated cell-type and tissue domain analysis that is readily applicable to numerous in situ transcriptomics methods. 


\section{Methods}

Using KDE to generate the gene expression vector field. We used the n-

dimensional $\mathrm{KDE}$ algorithm to estimate the density of mRNAs in $2 \mathrm{D}$ and 3D. To compute Gaussian KDE, we used our own implementation of the KDE algorithm for rapid computation. Spatial distribution of the probability of mRNA presence $\hat{p}$ is estimated using the kernel density estimation, as below Eq. (1);

$$
\hat{p}(\mathbf{x})=\frac{1}{N} \sum_{i=1}^{N} \kappa_{h}\left(\mathbf{x}-\mathbf{x}_{i}\right)
$$

where:

$\kappa_{h}$ : a kernel function with a bandwidth $h$

$N$ : the number of data points

$\mathbf{x}_{i}$ : location vector of the data point i (i.e., location of i-th mRNA)

Here we use the Gaussian kernel, as below Eq. (2):

$$
\kappa_{h}(\mathbf{x})=\frac{1}{\left(2 \pi h^{2}\right)^{\frac{d}{2}}} e^{-\frac{1}{2^{-}|x|^{2}}}
$$

where:

$\mathrm{h}$ : bandwidth of the Gaussian kernel

$d$ : dimension of the space where the data points reside ( 2 for $2 \mathrm{D}$, or 3 for $3 \mathrm{D}$ mRNA locations)

$\|\mathrm{x}\|$ : Euclidean norm (i.e., $\mathrm{L} 2$ norm) of vector $\mathrm{x}$

Note that the integration of $\hat{p}(\mathbf{x})$ all over the space is 1 . Therefore, the gene expression density is calculated by multiplying the number of mRNAs per gene to $\hat{p}$.

Calculation of spatial gene expression. The continuous estimation of gene expression density is discretized over pixels of the tissue image, which in our examples is set to a size of $1 \mu \mathrm{m}$. The expectation value of the estimated density in a unit pixel is approximated by multiplying the area of the unit pixel to the estimated gene expression density at the location of the pixel. Finally, we stack the estimated gene expression densities of genes to define the gene expression vector field over the image.

Selection of local maxima. Local maxima were selected based on the L1-norm of the vectors in the vector field, which is the total size of each vector in the image. For the selection algorithm, we used scikit-image Python package to select local maxima. Briefly, (1) maximum filter is applied to dilate the original image, (2) the locations where the maximum filtered image equal to the original image are selected. The maximum filter with size 3 was used throughout the examples presented in this paper.

Downsampling of the vector field. For a scalable cell-type identification analysis, the vector field is downsampled to a smaller set of vectors based on local maxima selection strategy (Supplementary discussion). SSAM applies two thresholds for local maxima selection: (1) a minimum expression threshold for a single gene defined as the height of a single Gaussian kernel to avoid regions with signal from only the Gaussian tail (see Discussion section for details), which also corresponds to the position of the observable drop in the histograms of gene expression (Supplementary Figs. 3A, 17A, 28A); (2) a minimum total gene expression (i.e. L1norm) threshold (Supplementary Figs. 3B, 17B, 28B). Furthermore, we implemented an optional 'input mask' feature to limit sampling of vectors to regions of the image containing informative data, e.g. a mask outlining the informative tissue area.

Comparison of local maxima and random sampling strategies. The two local maxima sampling methods, (1) local maxima sampling and (2) random downsampling, were compared to justify our preference of local maxima sampling method for the downstream analysis. The osmFISH data was used for the comparison. Firstly 11,469 local maxima vectors were found in the vector field using a window size of 3, a minimal gene expression and L1 norm thresholding. For comparison, the same number of vectors were randomly sampled from the vector field, using the same thresholds used for local maxima selection. At the locations of the vectors, both the local maxima and the random sampled locations, the classified cell types on the cell-type map guided by segmentation-based signatures are called. For each case, the Pearson's correlation coefficients between the vectors and the signature of the cell types are calculated and plotted as a distribution (Supplementary Fig. 39).

Variance stabilization of the vector field. Since the gene expression profiles of local maxima vectors are representative of the transcriptomes of cells, we considered them to be analogous to the gene expression count matrix obtained from single cell RNA sequencing (scRNA-seq) using unique molecular identifiers (UMI). Therefore, we normalized the local maxima vectors of the vector field (which would be representative of single cells) using sctransform ${ }^{37}$, a normalization and regularization algorithm for UMI count data. After that, each vector of the vector field is normalized using sctransform, with the same parameters previously used to normalize the local maxima.
Clustering of representative gene expression vectors. The SSAM framework supports clustering via DBSCAN ${ }^{24}$, HDBSCAN ${ }^{25}$, OPTICS ${ }^{26}$, and an implementation of the Louvain algorithm equivalent to that in the $\mathrm{R}$ package, Seurat ${ }^{27}$ DBSCAN, HDBSCAN, and OPTICS are implemented via the scikit-learn Python library. The Louvain clustering algorithm is based on the R package Seurat ${ }^{27}$ reimplemented in Python. In short, an SNN network with correlation metric is built using a python package NetworkX ${ }^{38}$. The weight of the network is calculated by a Jaccard similarity coefficient. A weight smaller than $1 / 15$ was set to zero. Clustering was done by detecting communities in the network using a Louvain community detection algorithm implemented in Python (python-louvain, https://python-louvain.readthedocs.io/). It is known that the Louvain algorithm is not sensitive in detecting small clusters ${ }^{39}$, optionally DBSCAN algorithm can be applied to subcluster each Louvain cluster. This sub-clustering strategy is conceptually similar to the 'Polished Louvain' algorithm in Zeisel et al. ${ }^{31}$.

Diagnostic plots. After unsupervised clustering of gene expression vectors, some clusters may need to be manually merged or discarded. SSAM supports merging of clusters based on correlation of gene expression profile, however in many cases manual inspection is needed to rule out any non-trivial issues. To guide this process, SSAM generates a cluster-wise 'diagnostic plot', which consists of four panels: (1) location of the clustered vectors on the tissue image, (2) the pixels classified to belong the cluster signature (the cluster centroid), (3) the mean expression profile of the clustered vectors, and (4) the t-SNE or UMAP embedding.

In the three datasets analyzed the clusters to be merged or removed often showed a discordance between the location of sampled vectors used to determine the cluster (panel 1) and the pixels classified to belong to that cluster (panel 2). In case of overclustering, i.e. when a cell-type signature is split over 2 clusters, the map typically does not classify the full shape of the cells but instead only fragments (panel 2), and having almost the same marker gene expression of another cluster (panel 3). Such clusters can be merged. For dubious clusters that should be removed, we observed that vectors usually originate from outside the tissue region or from image artifacts (panel 1), or that the gene expression does not show any clear expression of marker genes or similarity to expected gene expression profiles (panel 3).

The remaining clusters are then annotated by comparing cluster marker genes to known cell-type markers. Note that in many cases, the identity of clusters can be easily assigned by comparing the centroids of the clusters to the known cell-type signatures, e.g., from single cell RNA sequencing. To support rapid annotation of cell types to clusters, SSAM additionally shows the highest correlating known celltype signature should this data be available in panel 3. The diagnostic plots for osmFISH, MERFISH, and multiplexed smFISH data are available online in the Jupyter notebook uploaded to zenodo (https://doi.org/10.5281/zenodo.3478502) ${ }^{40}$

Statistical evaluation of cell-type mapping. The accuracy of the SSAM cell-type map was validated by comparing the published osmFISH segmentation and the SSAM de novo cell-type map by two different methods.

Firstly, to quantitatively compare concordance of cell-type we implemented a matching score. The matching score for any given cell type is defined as the number of segmented cells with at least $10 \%$ of matched with the SSAM guided or de novo mode cell-type map of the corresponding cell type of the segment, divided by the total number of segments of the cell type which represents the ratio of segments identified by SSAM. The threshold of $10 \%$ was empirically selected to account for differences in cell location in the tissue, especially for very small cells where subtle changes in cell-type labeling can drastically reduce the overlap within the segmented area.

Secondly, for evaluation of discrepancies in cell-type locations compared to the original studies, we compare the unique part of each segmentation and SSAM de novo cell-type map to the parts that are overlapping in both maps in the osmFISH dataset. The gene expression vectors originating from overlapping parts of the same cell types (Supplementary Table 3), were regarded as the ground truth set. Then, two sets of unique vectors were defined: (1) the segmentation-only set, the vectors from the regions occupied by segments excluding the overlap, and (2) the SSAMonly set, the vectors from SSAM cell-type map only regions. The distribution of the gene expression vectors in the overlapping set was then compared to the two unique parts (Supplementary Fig. 14A). To compare the accuracy of cell-type mapping of the two unique parts, Pearson's correlation coefficient is calculated between the mean expression of the ground truth set and the vectors in each set (Supplementary Fig. 14B).

Quantification of doublets. The doublet rates were evaluated by two Python packages, DoubletDetection ${ }^{41}$, and Scrublet ${ }^{42}$ (Supplementary Table 8 ). As the two algorithms require raw counts as input, the unnormalized raw vectors at local maxima used for clustering analysis were used as input of the two algorithms, as an analogy of the raw counts. For DoubletDetection, the doublet rate was calculated by dividing the number of doublets reported by the number of total local maxima. The doublet rate quantification by both methods was consistent, and negligible in the osmFISH and multiplexed smFISH datasets (average doublet rate of $<0.5 \%$ for both), and marginal for MERFISH (average doublet rate of $3 \%$ ). 
SSAM analysis of osmFISH data. KDE was performed with a bandwidth of $2.5 \mu \mathrm{m}$. The individual gene expression threshold and total gene expression threshold for selection of local maxima were 0.027 (the height of a single Gaussian) and 0.04 , respectively (Supplementary Fig. 3A, B). Since the selected local maxima includes many locations outside of the tissue area, we further filtered local maxima based on their local density approximated using the k-nearest neighbor algorithm. More specifically, local maxima with a density lower than 0.002 over the closest 100 local maxima, corresponding to fewer than 100 local maxima in a $126.2 \mu \mathrm{m}$ radius, were filtered out (Supplementary Fig. 3C). The selected local maxima vectors were passed to sctransform to determine normalization parameters, after which the whole vector field was normalized.

In SSAM guided mode, the mRNA count matrix of both the previously segmented cells and the scRNA-seq data were normalized by sctransform. The centroid of each of the annotated clusters was used to classify cell types in the vector field, generating a cell-type map guided by prior knowledge.

In SSAM de novo mode, the selected local maxima vectors were clustered using the Louvain algorithm with a resolution of 0.15 , resulting in 66 clusters (Supplementary Fig. 4A). Distinct clusters representing the same cell types were identified and then manually merged, and spurious clusters were removed, resulting in a total of 30 clusters (Fig. 2A, B). For each cluster, the vectors with insufficient correlation to its cluster medoid were excluded from the centroid calculation (Supplementary Fig. 1B). The cluster centroids were compared to that of the segmentation-based (Supplementary Fig. 4B) and scRNA-seq cell-type signatures (Supplementary Fig. 4C) using Pearson's correlation coefficient. The de novo clusters were named after the highest correlating segmentation-based cluster. Note that clusters closest mapped to Inhibitory IC and Inhibitory CP cell types do not only appear in the internal capsule and caudoputamen, but also in the cortex. Therefore, we renamed these clusters to Inhibitory Kcnip2 (since Kcnip2 was the third most expressed gene for this cluster) and Inhibitory Rest, respectively. After classification of the local maxima, we quantified the doublet rates (Methods, Supplementary Table 8)

Tissue domain analysis was performed using a sliding circular window with radius $100 \mu \mathrm{m}$ with a step of $10 \mu \mathrm{m}$. The cell-type proportions from each window were clustered using agglomerative hierarchical clustering with 15 clusters as an initial estimate, subsequently merging the clusters with correlation coefficients higher than 0.8 . Spatially connected clusters with a correlation coefficient higher than 0.6 were merged. The resulting domain map was resized to match the size of the cell-type map, after which the cells in different domains were colored.

Comparison of total gene expression between SSp cell types. The L5_All.loom loom object containing scRNA-seq expression data of half a million cells from the mouse nervous system ${ }^{31}$ was downloaded (http://mousebrain.org/downloads. html). The total number of mRNA molecules per cell were extracted and aggregated by their level 2 class labels (astrocytes, immune, vascular, ependymal, neuronal, peripheral glia and oligodendrocyte cells) using Python. The counts were log normalized and subsequently followed a normal distribution (tested using the Shapiro-Wilk test for normality, all $p$-values $<1 \times 10 \mathrm{e}-4$ for each class), therefore a Student's t-test was applicable. For each of the two classes of interest (astrocytes and immune), we performed independent log-space t-tests for unequal sample sizes and unequal variance against each of the other classes. Both astrocyte and immune cell classes have significantly lower mRNA molecule counts compared to other cell types (all p-values $<1 \times 10 \mathrm{e}-12$ ). While the distribution of mRNA counts in log space followed a normal distribution, the use of a Student's t-test for large numbers may be not appropriate. Hence, we also describe the difference in their distributions. For both astrocyte and immune cell classes, more than half of the cells of each class exhibited a lower UMI count than the lowest quartile of any other cell class. Furthermore, we quantified the effect size of the number of UMIs in astrocyte and immune cell types against others using Cohen's $\mathrm{d}$. The effect size of astrocyte cell types compared to other non-immune cell types combined was large (Cohen's $\mathrm{d}$ of 0.87 ), and large to huge for individual cell types (Cohen's $\mathrm{d}$ of 1.11, 2.47, 0.85, $2.01,1.77$ comparing astrocytes to vascular, ependymal, neuronal, peripheral glia and oligodendrocytes cell types, respectively). The effect size of immune cell types compared to other non-astrocyte cell types combined was medium (Cohen's d of 0.79 ), and medium to very large for individual cell types (Cohen's $\mathrm{d}$ of $0.81,1.73$, $0.74,1.59,1.47$ comparing immune cells to vascular, ependymal, neuronal, peripheral glia and oligodendrocytes cell types, respectively). Cohen's d indicated a small effect size between astrocyte and immune cell populations (0.21).

SSAM analysis of MERFISH data. KDE was performed with bandwidth $2.5 \mu \mathrm{m}$. Local maxima were filtered using a gene expression threshold of 0.0055 , and then filtered with total gene expression threshold of 0.0035 (Supplementary Fig. 17A, B). The selected local maxima vectors were passed to sctransform to determine normalization parameters, after which the whole vector field was normalized.

In SSAM guided mode, the mRNA count matrix of both the previously segmented cells and the scRNA-seq data were normalized by sctransform. The centroid of each of the annotated clusters was used to classify cell types in the vector field, generating a cell-type map guided by prior knowledge.

For SSAM de novo mode, the selected vectors were clustered using the Louvain algorithm with a resolution of 0.15 , resulting in 68 clusters (Supplementary Fig. 17C). By manual inspection of gene expression and localization, overclustering was merged, and spurious clusters were removed, resulting in a total of 50 clusters (Fig. 2A, B). For each cluster, the vectors that did not have high correlation to its cluster medoid were excluded from the centroid calculation (Supplementary Fig. 1B). The centroids of the clusters are compared with that of the segmentationbased clustering result and scRNA-seq result using Pearson's correlation coefficient (Supplementary Fig. 17E, F). The SSAM de novo clusters correlating best to inhibitory and excitatory neurons were named based on the most highly expressed gene of each cluster, and the non-neuronal clusters were named based on the previous study ${ }^{5}$. After classification of the local maxima, we quantified the doublet rates (Methods, Supplementary Table 8). We noticed a number of small blobs on the cell-type map, which are resultant from cells on a different plane in the $3 \mathrm{D}$ image (Supplementary Movie 2). After classification of the local maxima, we quantified the doublet rates (Methods, Supplementary Table 8),

Tissue domain analysis based on the cell-type map was performed using a sliding spherical window with radius $100 \mu \mathrm{m}$ with a step of $10 \mu \mathrm{m}$. The cell-type proportions from each window were clustered using agglomerative hierarchical clustering with 20 clusters as an initial estimate, subsequently merging the clusters with correlation coefficient higher than 0.8 . The resulting domain map was resized to match the size of the cell-type map, after which the cells in different domains were colored.

Comparison of neuronal cell type localization. For a number of inhibitory and excitatory neuronal subtypes identified in the posterior POA tissue image using SSAM de novo mode, we identified the best matching cell types based on Pearson correlation of their gene expression signatures (Supplementary Fig. 17F). We matched the following cell types: SSAM cluster 39 (C39) called Inhibitory Coch to Moffitt cluster I-12, C16 Inhibitory Arhgap36 to I-13, C45 Inhibitory Isr4 to I-15, C34 Inhibitory Calcr to I-14, C14 Inhibitory Gda to I-23, C19 Excitatory Cbln1Cbln2 to E-19, C42 Excitatory Omp to E-16, C25 Excitatory Necab1-Gda to E-9, C8 Excitatory Necab1 to E-14, and C36 Excitatory Col25a1 to E-24. For these cell types we checked the tissue localizations reported in the previous studies Figs. 5a, c, $\mathrm{e}, 6 \mathrm{~b}, \mathrm{~d}$, and $\mathrm{S} 17^{5}$. Side-by-side comparison of the localization of these neuronal cell types revealed very similar patterns of localization computed by SSAM and the original publication (Supplementary Fig. 22).

3D modeling of MERFISH cell-type maps. Firstly, the connected components in 3D were determined using the python package connected-components-3d (https:// github.com/seung-lab/connected-components-3d). Components comprising fewer than 100 voxels were removed. After this, the voxels filling connected components were removed, and only the contours were used for the vertex of the $3 \mathrm{D}$ models. For each vertex, the vertex normal was calculated by simple physics simulation, assuming that the direction of a vertex normal vector is the same as the force vector when there are pulling forces between all of the contour voxels. The surface of the objects was reconstructed using screened Poisson reconstruction algorithm ${ }^{43,44}$ using default parameters. The number of vertices was reduced to $5 \%$ of the total number of vertices using the 'vtkQuadricDecimation' function ${ }^{45,46}$ of VTK library ${ }^{47}$. Finally, the objects were merged into a single file. Each scene of the rotating movie was created using Meshlab ${ }^{48}$

VISp multiplexed smFISH data generation. Multiplexed smFISH data of the mouse primary visual cortex (VISp) was generated as part of the SpaceTx consortium. All procedures were carried out in accordance with Institutional Animal Care and Use Committee protocols at the Allen Institute for Brain Science. Animals were provided food and water ad libitum and were maintained on a regular 12-h day/night cycle at no more than five adult animals per cage.

Silanization of coverslips (\#1.5, Thorlabs CG15KH) was performed by plasma cleaning for $30 \mathrm{~min}$ in a Plasma-Prep III (SPI 11050-AB), followed by vapor deposition of 3-aminopropyltriethoxysilane (APES, Sigma A3648) in a vacuum for $10 \mathrm{~min}$. Coverslips were then washed in $100 \%$ methanol for $2 \times 5 \mathrm{~min}$, allowed to dry, and stored in a dust-free environment until use.

Fresh-frozen mouse brain tissue was sectioned at $10 \mu \mathrm{m}$ onto silanized coverslips, let dry for $20 \mathrm{~min}$ at $-20^{\circ} \mathrm{C}$, then fixed for $15 \mathrm{~min}$ at $4{ }^{\circ} \mathrm{C}$ in $4 \%$ PFA in PBS. Sections were washed $3 \times 10 \mathrm{~min}$ in PBS, then permeabilized and dehydrated with chilled $100 \%$ methanol at $-20^{\circ} \mathrm{C}$ for $10 \mathrm{~min}$ and allowed to dry. Sections were stored at $-80^{\circ} \mathrm{C}$ until use. Frozen sections were rehydrated in $2 \times$ SSC (Sigma 20XSSC, 15557036) for $5 \mathrm{~min}$, then treated 10 min with $8 \%$ SDS (Sigma 724255) in $\mathrm{PBS}$ at room temperature. Sections were washed 5 times in $2 \times$ SSC. Sections were then incubated in hybridization buffer (10\% Formamide (v/v, Sigma 4650$), 10 \%$ dextran sulfate (w/v, Sigma D8906), $200 \mu \mathrm{g} / \mathrm{mL}$ BSA (ThermoFisher AM2616), 2 $\mathrm{mM}$ ribonucleoside vanadyl complex (New England Biolabs S1402S), $1 \mathrm{mg} / \mathrm{ml}$ tRNA (Sigma 10109541001) in $2 \mathrm{X}$ SSC) for $5 \mathrm{~min}$ at $37^{\circ} \mathrm{C}$. Probes were diluted in hybridization buffer at a concentration of $250 \mathrm{nM}$ and hybridized at $37^{\circ} \mathrm{C}$ for $2 \mathrm{~h}$. Following hybridization, sections were washed $2 \times 10 \mathrm{~min}$ at $37^{\circ} \mathrm{C}$ in wash buffer ( $2 \times$ SSC, $20 \%$ Formamide), and $1 \times 10 \mathrm{~min}$ in wash buffer with $5 \mu \mathrm{g} / \mathrm{ml}$ DAPI (Sigma 32670), then washed 3 times with 2X SSC. Sections were then imaged in Imaging buffer $(20 \mathrm{mM}$ Tris- $\mathrm{HCl} \mathrm{pH} 8,50 \mathrm{mM} \mathrm{NaCl}, 0.8 \%$ glucose (Sigma G8270), $30 \mathrm{U} / \mathrm{ml}$ pyranose oxidase (Sigma P4234), $50 \mu \mathrm{g} / \mathrm{ml}$ catalase (Abcam ab219092). Following imaging, sections were incubated $3 \times 10 \mathrm{~min}$ in stripping buffer $\left(65 \%\right.$ formamide, $2 \times$ SSC) at $30^{\circ} \mathrm{C}$ to remove hybridization probes from the 
first round. Sections were then washed in $2 \times$ SSC for $3 \times 5 \mathrm{~min}$ at room temperature before repeating the hybridization procedure.

Multiplexed smFISH imaging was performed on an inverted epifluorescence microscope (Zeiss Axio Observer.Z1) with a $\times 63$ magnification, numerical aperture 1.4 oil immersion objective. A motorized $\mathrm{x}, \mathrm{y}$ stage with linear encoders and $\mathrm{z}$ piezo top-plate (Applied Scientific Instruments MS 2000-500) positioned the sample and performed $\mathrm{z}$ stacks. A $\mathrm{z}$ stack was collected in each color at each stage position to sample the $\mathrm{z}$ depth of the sample at $300 \mathrm{~nm}$ spacing. A high-speed filterwheel (Zeiss) directly below the dichroic turret filtered emission fluorescence before imaging with an sCMOS camera (Hamamatsu ORCA Flash4.0) (pixel size: 100 $\mathrm{nm}$ ). After each round of hybridization, images were collected using the same set of $\mathrm{x}, \mathrm{y}$ tile locations, with samples aligned manually based on DAPI fluorescence before each acquisition. Diffraction-limited smFISH spots were localized in 3D image stacks by finding local maxima after spatial bandpass filtering. A complete list of primers used is included in Supplementary Data 1 . The raw images can be downloaded via an Amazon S3 bucket (see Data Availability).

SSAM analysis of VISp multiplexed smFISH data. KDE was performed with bandwidth $2.5 \mu \mathrm{m}$. Local maxima were filtered using a gene expression threshold of 0.027 , and then filtered with total gene expression threshold of 0.2 (Supplementary Fig. 28A, B). The selected local maxima vectors were passed to sctransform to determine normalization parameters, after which the whole vector field was normalized. To identify rare cell types expected to exist in this tissue, the initial clustering result by Louvain algorithm was sub-clustered by DBSCAN (Method). Initially 49 clusters were obtained with a resolution parameter of 0.15 . By manual inspection, several over-clustered cell types, including nine L2/3 IT 1, two L2/3 IT 2, six L4 IT 2, six L6 CT, and two L6 IT 2 clusters were merged, and one spurious cluster was removed, resulting in 28 clusters. The centroids of the clusters are compared with that of scRNA-seq result using Pearson's correlation coefficient (Supplementary Fig. 28E). The clusters were named after the highest correlating scRNA-seq cluster, except the newly found 'L4 IT Superficial' (L4 IT 2) cluster. After classification of the local maxima, we quantified the doublet rates (Methods, Supplementary Table 8).

Tissue domains were defined using a sliding circular window with radius $100 \mu \mathrm{m}$ with step of $10 \mu \mathrm{m}$ over the cell-type map image. Cell type compositions of the windows were clustered using agglomerative clustering, initially with 20 clusters. Clusters with Pearson's correlation higher than 0.7 were merged to result in nine clusters. Further, two clusters were merged since they were different parts of the Pia layer, resulting in a final set of seven clusters representing tissue domains (Fig. 6).

Plotting. The python packages h5py 2.10.0, loompy 3.0.1, matplotlib 3.1.0 ${ }^{49}$, matplotlib-scalebar 0.6.0, seaborn $0.9 .0^{50}$, were used to draw $2 \mathrm{D}$ images, plots, and heatmaps. We include helper functions in SSAM to easily generate plots.

Movies. For 3D reconstruction the PoissonRecon command-line utility from Adaptive Multigrid Solvers 12.00, Meshlab 2016.12, vtk 8.1.2, and python-plyfile 0.7.1 were used. The supplementary movies were generated by using Virtualdub (1.10.4-AMD64, http://www.virtualdub.org/) and ffmpeg 3.4.4. The H.264 codec was used to compress videos.

Software. Python version 3.7.0 was used throughout. The following python packages were used:

networkX 2.3, numpy 1.17.1, scipy 1.3.1, pandas 0.25.1, matplotlib 3.1.0, seaborn 0.9.0, scikit-learn 0.21.3, umap-learn 0.3.10, python-louvain 0.13, sparse 0.8.0, scikit-image 0.15.0, pyarrow 0.15.1, packaging 20.8. R 3.6.0 package sctransform 0.2 .0 was used for normalization and variance stabilization of the data, requiring feather 0.3.3.

Reporting summary. Further information on research design is available in the Nature Research Reporting Summary linked to this article.

\section{Data availability}

All large files are available online from Zenodo (https://doi.org/10.5281/ zenodo. 3478502$)^{40}$

The osmFISH data (Codeluppi et al., 2018) used within the study is available from http://linnarssonlab.org/osmFISH/availability/. The single-cell RNA sequencing data of the mouse somatosensory cortex ${ }^{28,29}$ used for SSAM guided mode analysis are available from http://loom.linnarssonlab.org/. The single-cell RNA sequencing data ${ }^{31}$ used to compare total mRNA molecules between cell types are available from http://mousebrain. org/. The high-resolution poly-A and DAPI images of osmFISH data (Codeluppi et al., 2018) were kindly provided by Sten Linnarsson; the images are available by request due to the size of the image. The MERFISH data (Moffitt et al., 2018) is available from Dryad (https://datadryad.org/stash/dataset/doi:10.5061/dryad.8t8s248) ${ }^{51}$. Mouse VISp multiplexed smFISH spot data are available from Zenodo (https://doi.org/10.5281/ zenodo.3478502 $)^{40}$. The mouse VISp image data is available for download via an Amazon S3 bucket https://s3.amazonaws.com/starfish.data.spacetx/smFISH/mouse/ formatted_with_DAPI/experiment.json. The image is formatted in the SpaceTx format (https://spacetx-starfish.readthedocs.io/en/latest/help_and_reference/spacetx-format/ index.html?highlight=spacetx) and can be downloaded using the starfish python package (https://spacetx-starfish.readthedocs.io/) using the 'starfish.Experiment.from_json' command.

\section{Code availability}

The source code of SSAM is available online at Github (https://github.com/eilslabs/ssam) and Zenodo ${ }^{52}$. Three Jupyter notebooks (osmFISH, MERFISH, and multiplexed smFISH analyses) are deposited in a Github repository (https:/github.com/HiDiHlabs/ ssam_example), which outline the commands used to download and pre-process the data, used to reproduce the results and figures of this study. The Github repository also contains the extensive diagnostic plots used for parameter selection, and choice of removal or merging of clusters.

Received: 1 June 2020; Accepted: 14 May 2021; Published online: 10 June 2021

\section{References}

1. Svensson, V., Vento-Tormo, R. \& Teichmann, S. A. Exponential scaling of single-cell RNA-seq in the past decade. Nat. Protoc. 13, 599-604 (2018).

2. Regev, A. et al. Science forum: the human cell atlas. Elife 6, 27041 (2017).

3. Luecken, M. D. \& Theis, F. J. Current best practices in single-cell RNA-seq analysis: a tutorial. Mol. Syst. Biol. 15, e8746 (2019).

4. Salmén, F. et al. Multidimensional transcriptomics provides detailed information about immune cell distribution and identity in HER2+ breast tumors. bioRxiv 358937 (2018) https://doi.org/10.1101/358937.

5. Moffitt, J. R. et al. Molecular, spatial, and functional single-cell profiling of the hypothalamic preoptic region. Science 362, 5324 (2018).

6. Codeluppi, S., Borm, L. E., Zeisel, A. \& La Manno, G. Spatial organization of the somatosensory cortex revealed by osmFISH. Nat. Methods 15, 932-935 (2018).

7. Chen, K. H., Boettiger, A. N., Moffitt, J. R., Wang, S. \& Zhuang, X. Spatially resolved, highly multiplexed RNA profiling in single cells. Science 348, aaa6090 (2015).

8. Lubeck, E., Coskun, A. F., Zhiyentayev, T., Ahmad, M. \& Cai, L. Single-cell in situ RNA profiling by sequential hybridization. Nat. Methods 11, 360-361 (2014).

9. Ke, R. et al. In situ sequencing for RNA analysis in preserved tissue and cells. Nat. Methods 10, 857-860 (2013).

10. Lee, J. H. et al. Fluorescent in situ sequencing (FISSEQ) of RNA for gene expression profiling in intact cells and tissues. Nat. Protoc. 10, 442-458 (2015)

11. Wang, X. et al. Three-dimensional intact-tissue sequencing of single-cell transcriptional states. Science 361, 5691 (2018).

12. Ståhl, P. L. et al. Visualization and analysis of gene expression in tissue sections by spatial transcriptomics. Science 353, 78-82 (2016).

13. Maniatis, S. et al. Spatiotemporal dynamics of molecular pathology in amyotrophic lateral sclerosis. Science 364, 89-93 (2019).

14. Vickovic, S. et al. High-definition spatial transcriptomics for in situ tissue profiling. Nat. Methods 16, 987-990 (2019).

15. Hodneland, E., Kögel, T., Frei, D. M., Gerdes, H.-H. \& Lundervold, A. CellSegm - a MATLAB toolbox for high-throughput 3D cell segmentation. Source Code Biol. Med 8, 16 (2013).

16. Salvi, M. et al. Automated segmentation of fluorescence microscopy images for 3D cell detection in human-derived cardiospheres. Sci. Rep. 9, 6644 (2019)

17. Kong, J. et al. Automated cell segmentation with 3D fluorescence microscopy images. In 2015 IEEE 12th International Symposium on Biomedical Imaging (ISBI) 1212-1215 (2015).

18. Jiang, J., Kao, P.-Y., Belteton, S. A., Szymanski, D. B. \& Manjunath, B. S. Accurate 3D Cell Segmentation using Deep Feature and CRF Refinement. arXiv [cs.CV] (2019).

19. Shah, S., Lubeck, E., Zhou, W. \& Cai, L. In situ transcription profiling of single cells reveals spatial organization of cells in the mouse hippocampus. Neuron 92, 342-357 (2016).

20. Kishi, J. Y. et al. SABER amplifies FISH: enhanced multiplexed imaging of RNA and DNA in cells and tissues. Nat. Methods 16, 533-544 (2019).

21. Halpern, K. B. et al. Single-cell spatial reconstruction reveals global division of labour in the mammalian liver. Nature 542, 352-356 (2017).

22. Lignell, A., Kerosuo, L., Streichan, S. J., Cai, L. \& Bronner, M. E. Identification of a neural crest stem cell niche by Spatial Genomic Analysis. Nat. Commun. 8, 1830 (2017).

23. Thomas, R. M. \& John, J. A review on cell detection and segmentation in microscopic images. 1-5 (2017).

24. Ester, M., Kriegel, H.-P., Sander, J. \& Xu, X. A density-based algorithm for discovering clusters in large spatial databases with noise. In Proceedings of the 
Second International Conference on Knowledge Discovery and Data Mining 226-231 (AAAI Press, 1996).

25. McInnes, L., Healy, J. \& Astels, S. hdbscan: Hierarchical density based clustering. JOSS 2, 205 (2017).

26. Ankerst, M., Breunig, M. M., Kriegel, H.-P. \& Sander, J. OPTICS: ordering points to identify the clustering structure. SIGMOD Rec. 28, 49-60 (1999).

27. Butler, A., Hoffman, P., Smibert, P., Papalexi, E. \& Satija, R. Integrating singlecell transcriptomic data across different conditions, technologies, and species. Nat. Biotechnol. 36, 411-420 (2018).

28. Zeisel, A. et al. Cell types in the mouse cortex and hippocampus revealed by single-cell RNA-seq. Science 347, 1138-1142 (2015).

29. Marques, S. et al. Oligodendrocyte heterogeneity in the mouse juvenile and adult central nervous system. Science 352, 1326-1329 (2016).

30. Tasic, B. et al. Shared and distinct transcriptomic cell types across neocortical areas. Nature 563, 72-78 (2018)

31. Zeisel, A. et al. Molecular architecture of the mouse nervous system. Cell 174, 999-1014.e22 (2018).

32. Perkel, J. M. Starfish enterprise: finding RNA patterns in single cells. Nature 572, 549-551 (2019).

33. Tasic, B. et al. Adult mouse cortical cell taxonomy revealed by single cell transcriptomics. Nat. Neurosci. 19, 335-346 (2016).

34. Tomioka, R. et al. Demonstration of long-range GABAergic connections distributed throughout the mouse neocortex. Eur. J. Neurosci. 21, 1587-1600 (2005).

35. Gerashchenko, D. et al. Identification of a population of sleep-active cerebral cortex neurons. Proc. Natl Acad. Sci. USA 105, 10227-10232 (2008).

36. Dries, R. et al. Giotto: a toolbox for integrative analysis and visualization of spatial expression data. Genome biol. 22, 78 (2021).

37. Hafemeister, C. \& Satija, R. Normalization and variance stabilization of singlecell RNA-seq data using regularized negative binomial regression. Genome Biol. 20, 296 (2019).

38. Hagberg, A., Swart, P. \& S Chult, D. Exploring network structure, dynamics, and function using NetworkX. In Proceedings of the 7th Python in Science Conference (SciPy2008) 11-15 (Los Alamos National Lab.(LANL), Los Alamos, NM (United States), 2008).

39. Fortunato, S. \& Barthélemy, M. Resolution limit in community detection. Proc. Natl Acad. Sci. USA 104, 36-41 (2007).

40. Park, J. et al. Supplemental Data for: Segmentation-free inference of cell types from in situ transcriptomics data. Zenodo (2019) https://doi.org/10.5281/ zenodo.3478502.

41. Gayoso, A., Shor, J., Carr, A. J., Sharma, R. \& Pe'er, D. JonathanShor/ DoubletDetection: HOTFIX: Correct setup.py installation. Zenodo (2019). https://doi.org/10.5281/zenodo.3376859

42. Wolock, S. L., Lopez, R. \& Klein, A. M. Scrublet: computational identification of cell doublets in single-cell transcriptomic data. Cell Syst. 8, 281-291.e9 (2019).

43. Kazhdan, M., Bolitho, M. \& Hoppe, H. Poisson surface reconstruction. In Proceedings of the fourth Eurographics symposium on Geometry processing 61-70 (2006).

44. Kazhdan, M. \& Hoppe, H. Screened poisson surface reconstruction. ACM Trans. Graph. 32, 29 (2013).

45. Garland, M. \& Heckbert, P. S. Surface simplification using quadric error metrics. In Proceedings of the 24th annual conference on Computer graphics and interactive techniques 209-216 (ACM Press/Addison-Wesley Publishing Co., 1997).

46. Hoppe, H. New quadric metric for simplifying meshes with appearance attributes. in Proceedings Visualization '99 (Cat. No.99CB37067) 59-510 (1999).

47. Schroeder, W., Martin, K. \& Lorensen, B. The Visualization Toolkit: An Object-oriented Approach to 3D Graphics. (Kitware, 2006).

48. Cignoni, P. et al. Meshlab: an open-source mesh processing tool. Eurographics Ital. chapter Conf. 2008, 129-136 (2008).

49. Caswell, T. A. et al. matplotlib/matplotlib v3.1.0. Zenodo (2019). https://doi. org/10.5281/zenodo.2893252.

50. Waskom, M. et al. mwaskom/seaborn: v0.9.0 (July 2018). Zenodo (2018). https://doi.org/10.5281/zenodo.1313201.
51. Moffitt, J. R. et al. Data from: Molecular, spatial and functional single-cell profiling of the hypothalamic preoptic region. Dryad (2018) https://doi.org/ 10.5061 /dryad. 8 t 8 s 248

52. Park, J. et al. SSAM 1.0.2. Zenodo (2021). https://doi.org/10.5281/zenodo.4721907.

\section{Acknowledgements}

We thank Sten Linnarsson and Jeffrey Moffitt for providing support and access to the osmFISH and MERFISH datasets, respectively. We also thank Yue Zhuo, Ed Lein, Jeremy Miller, Ambrose Carr, Nagarajan Paramasivam, Stephen Krämer, Zuguang Gu, Daniel Hübschmann, Luca Tosti, and Christian Conrad for helpful discussions and comments on data analysis. The authors also thank Bianca Hennig for designing Fig. 1, and assistance in improving figures. The preliminary analysis of multiplexed smFISH data occurred during the SpaceTx SpaceJam Hackathon at the Allen Institute for Brain Science, which was organized by Ed Lein, and generously supported by the Chan Zuckerberg Initiative. This publication is part of the Human Cell Atlas - www.humancellatlas org/publications. This research has received funding from the European Commission EU Horizon 2020 research and innovation program (ESPACE, 874710; EASI-Genomics, 824110; HCA Organoid, 874769). The authors acknowledge the financial support by the Federal Ministry of Education and Research of Germany in the framework of SAGE (project number 031L0265).

\section{Author contributions}

J.P., W.C. designed the concept and idea of S.S.A.M. J.P., W.C., R.E., N.I. conceived the study. B.T., E.G., T.N.N., B.L. acquired and interpreted the multiplexed smFISH data. J.P., W.C., S.T., T.N.N., N.I. performed data analysis. L.E.B., S.C., M.S., B.L., B.T., T.G., O.S. provided critical comments and discussions. R.E., N.I. supervised the study. All authors commented on and critically revised the manuscript.

\section{Funding}

Open Access funding enabled and organized by Projekt DEAL.

\section{Competing interests}

The authors declare no competing interests.

\section{Additional information}

Supplementary information The online version contains supplementary material available at https://doi.org/10.1038/s41467-021-23807-4.

Correspondence and requests for materials should be addressed to R.E. or N.I.

Peer review information Nature Communications thanks the anonymous reviewer(s) for their contribution to the peer review of this work.

Reprints and permission information is available at http://www.nature.com/reprints

Publisher's note Springer Nature remains neutral with regard to jurisdictional claims in published maps and institutional affiliations.

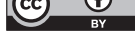

Open Access This article is licensed under a Creative Commons Attribution 4.0 International License, which permits use, sharing, adaptation, distribution and reproduction in any medium or format, as long as you give appropriate credit to the original author(s) and the source, provide a link to the Creative Commons license, and indicate if changes were made. The images or other third party material in this article are included in the article's Creative Commons license, unless indicated otherwise in a credit line to the material. If material is not included in the article's Creative Commons license and your intended use is not permitted by statutory regulation or exceeds the permitted use, you will need to obtain permission directly from the copyright holder. To view a copy of this license, visit http://creativecommons.org/ licenses/by/4.0/.

(C) The Author(s) 2021, corrected publication 2021 